\title{
Time-filtered Leapfrog Integration of Maxwell Equations using Unstaggered Temporal Grids
}

\author{
A. Mahalov, M. Moustaoui* \\ School of Mathematical and Statistical Sciences, Arizona State University, \\ Tempe, AZ 85287-1804
}

\begin{abstract}
A finite-difference time-domain method for integration of Maxwell equations is presented. The computational algorithm is based on the leapfrog time stepping scheme with unstaggered temporal grids. It uses a fourth-order implicit time filter that reduces computational modes and fourth-order finite difference approximations for spatial derivatives. The method can be applied within both staggered and collocated spatial grids. It has the advantage of allowing explicit treatment of terms involving electric current density and application of selective numerical smoothing which can be used to smooth out errors generated by finite differencing. In addition, the method does not require iteration of the electric constitutive relation in nonlinear electromagnetic propagation problems. The numerical method is shown to be effective and stable when employed within Perfectly Matched Layers (PML). Stability analysis demonstrate that the proposed method is effective in stabilizing and controlling numerical instabilities of computational modes arising in wave propagation problems with physical damping and artificial smoothing terms while maintaining higher accuracy for the physical modes. Comparison of simulation results obtained from the proposed method and those computed by the classical time
\end{abstract}

Preprint submitted to Journal of Computational Physics

10 August 2016

(C) 2016. This manuscript version is made available under the Elsevier user license http://www.elsevier.com/open-access/userlicense/1.0/ 
filtered leapfrog, where Maxwell equations are integrated for a lossy medium, within PML regions and for Kerr-nonlinear media show that the proposed method is robust and accurate. The performance of the computational algorithm is also verified by analyzing parametric four wave mixing in an optical nonlinear Kerr medium. The algorithm is found to accurately predict frequencies and amplitudes of nonlinearly converted waves under realistic conditions proposed in the literature.

Key words: Maxwell's equations, FDTD schemes, unstaggered leapfrog, time-filtered integration, reduction of dispersion errors.

* Corresponding author.

Email addresses: mahalov@asu.edu (A. Mahalov), mohamed.moustaoui@asu.edu (M. Moustaoui). 


\section{Introduction}

There are several numerical methods that are used to integrate Maxwell equations. The Finite-Difference Time-Domain (FDTD) technique refers to a finite difference approximation of Faraday's and Ampere's laws using a leapfrog scheme combined with centered finite difference approximations for spatial derivatives. This method was introduced for the first time by Yee [1]. It is the most common time-domain method used to numerically solve Maxwell's equations [2]. The FDTD method is an explicit leapfrog finite difference scheme. It employs central differences on a staggered grid in both time and space. The Yee scheme has the advantage of low complexity, generality, and simplicity for parallel computing. It is a flexible and simple method to implement for solving complex electromagnetic problems. The method requires one function evaluation per time step and can model electromagnetic responses of inhomogeneous materials with curved geometry $[3,4]$. However, the standard Yee method can introduce errors due to significant numerical dispersion induced by poor representation of spatial finite differences. Other FDTD schemes utilizing the same time stepping as in the Yee's method have been proposed. These schemes use a fourth-order approximations of spatial differences [5-12]. The fourth-order staggered difference achieves much lower numerical dispersion compared to the second-order spatial differencing.

A comparison of the accuracy of several low-dispersion FDTD schemes was analyzed by Shlager and Schneider [13]. They showed that almost all the schemes using high-order finite difference provide substantial improvement in the dispersion errors compared with the classical Yee algorithm. Finkelstein and Kastner [17] presented a methodology for deriving a dispersion reduction scheme based on modifications of the characteristic equation for electromag- 
netic wave propagation problems.

The schemes described above use different finite difference approximations, but they all use the same leapfrog time stepping method as in the Yee scheme. In these schemes, the temporal grid is staggered in that, the electric field $(E)$ is defined at $t_{n}=n \Delta t$ and $t_{n+1}=(n+1) \Delta t$ while the magnetic field $(H)$ is defined half time step away from $E$ at $t_{n-1 / 2}=(n-1 / 2) \Delta t$ and $t_{n+1 / 2}=(n+1 / 2) \Delta t$. Although temporal staggering is more accurate and suitable for the terms $\nabla \times \mathbf{H}$ and $\nabla \times \mathbf{E}$, it is incompatible with the timedifferencing needed to integrate other physical and artificial terms in the governing equations such as Perfectly Matched Layers (PML) [14,15] and selective numerical smoothing terms. For instance, since the electric field $E$ is not defined at $t_{n+1 / 2}=(n+1 / 2) \Delta t$, the time-staggered leapfrog formulation is usually combined with a semi-implicit trapezoidal scheme, which is employed to stably integrate terms representing current density $J=\sigma E$ and PML layers. In addition, applications of staggered temporal grids to Maxwell equations require solving a constitutive relation for the electric field at each time step in nonlinear electromagnetic propagation problems [16]. For similar reasons, the temporal staggered leapfrog method cannot be applied to electric and magnetic numerical smoothing terms. These artificial terms are traditionally introduced in the governing equations to reduce short oscillations generated by poor representation of spatial differencing.

In this paper we present a FDTD numerical algorithm for Maxwell equations using unstaggered temporal grids. It is based on a time-filtered leapfrog scheme using a fourth-order implicit time filter recently proposed in [20]. In this method, the electric and the magnetic fields are defined at the same temporal location $\left(t_{n}=n \Delta t\right)$. We will show that this method allows explicit 
integration of current density terms. It can be applied for numerical smoothers to control short oscillations inherent in the numerical solutions when centered differencing is used; and can also be employed to effectively and stably integrate PML terms. We will also demonstrate that this method does not require solving the electric constitutive relation in nonlinear electromagnetic propagation problems.

The time-unstaggered leapfrog is very simple to implement. This method is suitable for numerical integration of wave propagation problems. However, it suffers from a serious defect related to the development of spurious modes ( $2 \Delta t$ waves). These modes are neutral and do not interact with the physical solution in linear wave propagation. Nevertheless, they may become unstable for nonlinear problems and are unconditionally unstable when numerical smoothing, physical or artificial damping are employed.

The unphysical modes can be reduced by applying the standard RobertAsselin filtered leapfrog method [21,22], which uses a second-order time filter. However, the method introduces errors in the amplitude of the physical modes and degrades the accuracy of the solution to first order [26]. Alternative time stepping methods, such as high-order Runge-Kutta schemes, that do not have this defect have been described in the literature. These have been used in computational fluid dynamics (e.g., [24-28]) and Maxwell equations (e.g.. $[18,19])$. The advantage of the third-order Runge-Kutta scheme (RK3) is its conditional stability for both advection and diffusion equations. It is also stable for both even and odd-order finite difference approximations for the first derivative in advection problems [24]. RK3 has higher accuracy but it requires three function evaluations per time step. Traditionally, poorly resolved waves generated by finite differencing can be reduced by applying selective numeri- 
cal smoothers. The unfiltered leapfrog is unconditionally unstable when these smoothers are added to the equations.

The scheme proposed by Moustaoui et. al [20] produces third-order accuracy for the amplitude of the physical solution. It is superior compared with those based on second-order time filtering. Stability analysis of this scheme using the oscillation equation demonstrated that it is effective in reducing the computational modes. However, the behavior of this scheme and its stability when it is combined with spatial differencing and applied to Maxwell equations were not studied. In this paper, we propose a method based on a filtered leapfrog scheme using a staggered temporal grid, which can be employed to integrate Maxwell equations. The scheme can be applied in combination with both staggered and collocated spatial grids. We show that this method can be used explicitly to integrate terms representing current density, numerical smoothing and PML layers, without having the need to combine it with other time-stepping schemes. We also show that the method is effective for nonlinear electromagnetic propagation problems. The paper is organized as follows. The formulation of the scheme is presented in Section 2. Tests and stability analysis are shown in section 3. Applications for lossy material, PML layers and parametric four wave mixing in an optical nonlinear Kerr medium are demonstrated in Section 4. Finally a summary is given in section 5 .

\section{Formulation}

The time dependent components of Maxwell equations considered in this paper for an electromagnetic field propagation are the Faraday's and Ampere's laws. Assuming a linear, isotropic, nondispersive and lossy medium, these equations 
have the form:

$$
\begin{aligned}
& \frac{\partial \mathbf{D}}{\partial t}=\nabla \times \mathbf{H}-\mathbf{J} \\
& \frac{\partial \mathbf{B}}{\partial t}=-\nabla \times \mathbf{E}
\end{aligned}
$$

together with the linear material laws $\mathbf{B}=\mu \mathbf{H}, \mathbf{J}=\sigma \mathbf{E}, \mathbf{D}=\epsilon \mathbf{E}$.

In these equations, $\mathbf{E}$ is the electric field, $\mathbf{D}$ is the electric flux density, $\mathbf{H}$ is the magnetic field, $\mathbf{B}$ is the magnetic flux density, $\mathbf{J}$ is the electric current density, $\mu$ is the permeability, $\epsilon$ is the permittivity and $\sigma$ is the electric losses of the isotropic medium. The speed of light in the medium is $c=1 / \sqrt{\epsilon \mu}$.

A finite-difference time-domain method for integration of Maxwell equations developed in this paper uses time discretization of Eqs.1-2 with a modified unstaggered leapfrog time stepping scheme where the electric and magnetic fields are defined at the same location in time. It has the form:

$$
\begin{aligned}
& \frac{\mathbf{D}^{n+1}-\overline{\mathbf{D}}^{n-1}}{2 \Delta t}=\nabla \times \mathbf{H}^{n}-\mathbf{J}^{n}+\mathbf{P}_{E}^{n} \\
& \frac{\mathbf{B}^{n+1}-\overline{\mathbf{B}}^{n-1}}{2 \Delta t}=-\nabla \times \mathbf{E}^{n}+\mathbf{P}_{B}^{n},
\end{aligned}
$$

Here, $\mathbf{P}_{E}^{n}$ and $\mathbf{P}_{B}^{n}$ include terms representing absorbing Perfectly Matched Layers (PML) and contributions from numerical smoothers. The fields $\overline{\mathbf{D}}^{n-1}$ and $\overline{\mathbf{B}}^{n-1}$ are the solutions at $t=(n-1) \Delta t$ after applying a fourth-order time filter using a real constant $\gamma$ that determines the strength of the filter. These fields are implicitly determined in this numerical scheme as they depend on the unfiltered values of $\mathbf{D}^{n+1}$ and $\mathbf{B}^{n+1}$ : 


$$
\begin{aligned}
& \overline{\mathbf{D}}^{n-1}=\mathbf{D}^{n-1}+\gamma\left(-\overline{\mathbf{D}}^{n-3}+4 \overline{\mathbf{D}}^{n-2}-6 \overline{\mathbf{D}}^{n-1}+4 \mathbf{D}^{n}-\mathbf{D}^{n+1}\right) \\
& \overline{\mathbf{B}}^{n-1}=\mathbf{B}^{n-1}+\gamma\left(-\overline{\mathbf{B}}^{n-3}+4 \overline{\mathbf{B}}^{n-2}-6 \overline{\mathbf{B}}^{n-1}+4 \mathbf{B}^{n}-\mathbf{B}^{n+1}\right)
\end{aligned}
$$

Although the scheme uses an implicit time filter, it can be easily formulated recursively in an explicit manner. For instance, the formulation for $\mathbf{D}$ is as follows:

$$
\begin{aligned}
\overline{\mathbf{D}}^{n-1} & =\frac{-2 \Delta t \gamma \mathbf{F}^{n}+\tilde{\mathbf{D}}^{n-1}}{1+7 \gamma} \\
\mathbf{D}^{n+1} & =\frac{(1+6 \gamma) 2 \Delta t \mathbf{F}^{n}+\tilde{\mathbf{D}}^{n-1}}{1+7 \gamma} \\
\tilde{\mathbf{D}}^{n} & =\mathbf{D}^{n}+\gamma\left(-\overline{\mathbf{D}}^{n-2}+4 \overline{\mathbf{D}}^{n-1}+4 \mathbf{D}^{n+1}\right) .
\end{aligned}
$$

where $\mathbf{F}^{n}=\nabla \times \mathbf{H}^{n}-\mathbf{J}^{n}+\mathbf{P}_{E}^{n}$. The formulation for $\mathbf{B}$ is the same as for $\mathbf{D}$ except that $\mathbf{F}^{n}$ is given by the right hand side of Eq.4.

The unfiltered time-unstaggered leapfrog scheme is nondissipative, very simple to implement and requires only one function evaluation per time step. However, it is well known that this scheme generates high frequency $(2 \Delta t)$ computational modes. The Leapfrog method is well suitable for linear propagation problems that do not include physical or artificial damping terms (i.e current and hyper diffusion). For these problems the computational and physical modes are decoupled and do not interact with each other. These computational modes become a serious problem for nonlinear computations and for problems involving physical or artificial damping for which the computational modes are unconditionally unstable. These terms are the electric current density for lossy materials, the PML layer and numerical smoothing terms that can be introduced in Maxwell equations to reduce errors generated by spatial discretizations in the numerical approximation to the solution. The computa- 
tional modes are traditionally reduced by applying the Robert-Asselin time filter which uses a second-order explicit time filter:

$$
\overline{\mathbf{D}}^{n-1}=\mathbf{D}^{n-1}+\gamma_{2}\left(\overline{\mathbf{D}}^{n-2}-2 \mathbf{D}^{n-1}+\mathbf{D}^{n}\right)
$$

where $\gamma_{2}$ is the filter coefficient. However, this method reduces the accuracy of the amplitude of the physical solution to first-order. The implicit time filter used in Eqs.5-6 has the advantage of damping the computational modes while maintaining a third-order accuracy for the amplitude of the physical modes [20]. It also has the benefit of stabilizing the physical or artificial damping terms. Next, we analyze the stability of the proposed scheme using the one-dimensional Maxwell equations in combination with fourth-order finitedifference approximations evaluated on both staggered or collocated spatial grids.

\section{Wave propagation tests and stability analysis}

\subsection{Wave propagation tests}

Consider the one-dimensional discretized Maxwell equations :

$$
\begin{aligned}
& \frac{E_{i}^{n+1}-\bar{E}_{i}^{n-1}}{2 \Delta t}=\frac{1}{\epsilon} \frac{H_{i+1 / 2}^{a v}-H_{i-1 / 2}^{a v}}{\Delta x}+\nu\left(\frac{\partial^{4} E}{\partial x^{4}}\right)_{i}^{n} \\
& \frac{H_{p}^{n+1}-\bar{H}_{p}^{n-1}}{2 \Delta t}=\frac{1}{\mu} \frac{E_{p+1 / 2}^{a v}-E_{p-1 / 2}^{a v}}{\Delta x}+\nu\left(\frac{\partial^{4} H}{\partial x^{4}}\right)_{p}^{n},
\end{aligned}
$$

where $i$ and $p$ denote the locations on the grid of the electric and magnetic

fields respectively. These locations are related by $p=i+1 / 2$ for a staggered 
grid and $p=i$ for a collocated grid. The fields $H_{i+1 / 2}^{a v}$ and $E_{p+1 / 2}^{a v}$ represent some suitable spatial averaging of $E^{n}$ and $H^{n}$, which has the effect of producing a fourth-order accurate finite difference approximations for the spatial first derivatives.

For a staggered spatial grid, these averages have the forms:

$$
\begin{aligned}
& H_{i+1 / 2}^{a v}=\frac{-H_{p-1}^{n}+26 H_{p}^{n}-H_{p+1}^{n}}{24} \\
& E_{p-1 / 2}^{a v}=\frac{-E_{i-1}^{n}+26 E_{i}^{n}-E_{i+1}^{n}}{24} .
\end{aligned}
$$

They are derived by using Taylor series expansions and by writing the resulting finite difference approximations of the first derivatives in flux forms. For a staggered grid $(p=i+1 / 2)$, we have

$$
\begin{aligned}
\frac{H_{i+1 / 2}^{a v}-H_{i-1 / 2}^{a v}}{\Delta x} & =\frac{27\left(H_{p}-H_{p-1}\right)-\left(H_{p+1}-H_{p-2}\right)}{24 \Delta x} \\
& =\frac{27\left(H_{i+1 / 2}-H_{i-1 / 2}\right)-\left(H_{i+3 / 2}-H_{i-3 / 2}\right)}{24 \Delta x}
\end{aligned}
$$

which is the fourth-order approximation for the first spatial derivative.

For a collocated grid $(p=i), E^{a v}$ and $H^{a v}$ have the same form given by:

$$
H_{i+1 / 2}^{a v}=\frac{7\left(H_{i+1}^{n}+H_{i}^{n}\right)-\left(H_{i+2}^{n}+H_{i-1}^{n}\right)}{12}
$$

and the fourth-order approximation for the first derivative on this grid is:

$$
\frac{H_{i+1 / 2}^{a v}-H_{i-1 / 2}^{a v}}{\Delta x}=\frac{8\left(H_{i+1}-H_{i-1}\right)-\left(H_{i+2}-H_{i-2}\right)}{12 \Delta x} .
$$


The second terms in the rhs of Eqs. 11-12 represent hyper-diffusion. These terms are introduced as a fourth-order numerical smoother with coefficient $\nu$. This selective smoother is applied to smooth out the poorly resolved high wavenumber oscillations. These may be generated by the centered finite difference approximations of the spatial derivative. The numerical smoother term is approximated by:

$$
\left(\frac{\partial^{4} E}{\partial x^{4}}\right)_{i}=\frac{-E_{i-2}^{n}+4 E_{i-1}^{n}-6 E_{i}^{n}+4 E_{i+1}^{n}-E_{i+2}^{n}}{(\Delta x)^{4}}
$$

Next, we solve Eqs. 11-12 by using the unfiltered leapfrog (LF1), the filtered leapfrog proposed in this paper (LF2) and the one that uses the classical Robert-Asselin time filter (LF3). The numerical approximations obtained from these schemes are compared to the solution computed by RK3. LF2 uses a fourth-order implicit time filter with a coefficient of $\gamma=0.1$. The value of the filter strength $\gamma_{2}$ in Eq.10 used for LF3 is different. It is chosen according to the relation $\gamma_{2}=4 \gamma /(1+7 \gamma)[20]$. This choice ensures that as $k \Delta x$ approaches 0, the filtered schemes LF2 and LF3 damp the computational $2 \Delta t$ modes at the same rate.

The one-dimensional domain uses 101 grid points in the interval $0<x<100$. Periodic boundary conditions are imposed and the initial conditions consist of a half sine pulse that has the form:

$$
\begin{aligned}
& E^{0}(x)=\sin \left(\pi \frac{x-43}{14}\right), \text { for } \quad 43 \leq x \leq 57, \\
& E^{0}(x)=0, \text { otherwise; and } H^{0}(x)=0 .
\end{aligned}
$$

Equations 11 and 12 are numerically integrated within the domain using two 
choices of time step $\Delta t$ and grid spacing $\Delta x$ with corresponding Courant numbers $C r=\frac{1}{\sqrt{\epsilon \mu}} \frac{\Delta t}{\Delta x}$ of 0.5 and 0.3 . The total number of time steps is chosen such that the waves generated by the initial pulse are transported one revolution around the domain. Figure 1a superimposes the numerical approximations of the solution for the electric field obtained from the LF1, LF2 and LF3 schemes using a Courant number of $\mathrm{Cr}=0.5$. For comparison, the numerical approximations computed by the RK3 scheme are also superimposed in Fig.1. These approximations use a collocated spatial grid. The finite difference approximations are fourth-order accurate. LF2 and LF3 use a numerical smoother with a value of $s=\nu \Delta t / \Delta x^{4}=0.004$. The smoother is not included in LF1 $(\nu=0)$ because the computational modes are unconditionally unstable when the numerical smoother is applied to the unfiltered leapfrog scheme. The solution computed from LF1 produces short oscillations that have smaller amplitudes and higher wave numbers. In contrast to LF1, the LF2 and LF3 schemes are stable for both wave propagation and numerical smoothing contributions. The oscillations with high wavenumbers produced by the finite difference approximations are reduced in these schemes compared to the unfiltered leapfrog (LF1). However, the solution obtained from LF2 is much more accurate and is superior to the one computed from LF3. The traditional second-order time filter used in LF3 removes and stabilizes the computational modes but produces excessive dissipation resulting in a degradation of the physical solution. The solution obtained from LF2 is closer to the solution computed by RK3, which is also superimposed in Fig.1.

Figure 1b shows the solutions for the electric field approximated with a Courant number of $\mathrm{Cr}=0.3$. The solution calculated by LF1 is similar to the corresponding one that is presented in Fig.1a, with generation of short oscillations. This indicates that reducing the Courant number does not improve the numer- 
ical solutions obtained by LF1 in this case. The short oscillations are reduced in both LF2 and LF3. However, LF3 produces excessive damping of the physical solution compared to LF2.

The proposed scheme can also be used in combination with a staggered spatial grid. The numerical solutions obtained in this case are presented in Fig. 2 for a Courant number of $C r=0.3$. As in the collocated grid, the approximations obtained from LF2 are superior to those computed by LF1 and LF3. The short oscillations are reduced while the physical solution is simulated with higher accuracy. The numerical solution obtained from the Yee time stepping scheme combined with fourth-order approximations for the spatial derivatives (LF4) is also superimposed in Fig. 2. We note that while LF4 has high accuracy for the physical mode, it also generates oscillations with short wavelengths. The comparisons presented in Fig.1 demonstrate the ability of LF2 to stabilize the leapfrog scheme by controlling the computational modes in wave propagation problems including numerical smoothing contributions for which the unfiltered leapfrog is unconditionally unstable.

\subsection{Stability analysis}

Next, we examine the behavior of LF2 when applied to Eqs. 11-12 using fourthorder space differencing within both staggered and collocated grids. For this purpose, stability analysis is performed with $E$ and $H$ in the forms:

$$
E_{i}^{n}=\psi^{n} e^{j k i \Delta x} \text { and } H_{p}^{n}=\phi^{n} e^{j k p \Delta x}
$$

Here, $k$ is a real spatial wave number, $p=i$ for a collocated grid and $p=i+1 / 2$ for a staggered grid. $\psi^{n}$ and $\phi^{n}$ are complex functions, $j=\sqrt{-1}$ and $\Delta x$ is 
the grid spacing. Using these forms, Eqs.11-12 become

$$
\begin{aligned}
\psi^{n+1}-\bar{\psi}^{n-1} & =j \frac{2}{\epsilon} \frac{\Delta t}{\Delta x} \lambda \phi^{n}-2 s \eta \psi^{n} \\
\phi^{n+1}-\bar{\phi}^{n-1} & =j \frac{2}{\mu} \frac{\Delta t}{\Delta x} \lambda \psi^{n}-2 s \eta \phi^{n} .
\end{aligned}
$$

The function $\lambda$ is given by $\lambda(k \Delta x)=(8 \sin (k \Delta x)-\sin (2 k \Delta x)) / 6$ for a collocated grid, and $\lambda(k \Delta x)=(27 \sin (k \Delta x / 2)-\sin (3 k \Delta x / 2)) / 12$ for a staggered grid. The function $\eta=\eta(k \Delta x)=4(1-\cos (k \Delta x))^{2}$ for both grids, and $s=\nu \Delta t / \Delta x^{4}$. If we apply the time filter expressed in Eqs.5-6, use the amplification $A$ defined by $\psi^{n+1}=A \psi^{n}$ and $\phi^{n+1}=A \phi^{n}$, and set $\psi^{n-3}=\psi$ and $\phi^{n-3}=\phi$, then, Eqs.20-21 and the filter relations become:

$$
\begin{aligned}
\left(A^{2}+2 s \eta A\right) \psi-\bar{\psi} & =j \frac{2}{\epsilon} \frac{\Delta t}{\Delta x} \lambda A \phi \\
\left(A^{2}+2 s \eta A\right) \phi-\bar{\phi} & =j \frac{2}{\mu} \frac{\Delta t}{\Delta x} \lambda A \psi \\
\left(A^{2}+\gamma\left(1-4 A+6 A^{2}\right)\right) \bar{\psi} & =\left(A^{2}+\gamma\left(4 A^{3}-A^{4}\right)\right) \psi \\
\left(A^{2}+\gamma\left(1-4 A+6 A^{2}\right)\right) \bar{\phi} & =\left(A^{2}+\gamma\left(4 A^{3}-A^{4}\right)\right) \phi
\end{aligned}
$$

Eqs.22-25 can be combined to obtain the following equation:

$$
\left(\alpha_{1}\left(A^{2}+2 s \eta A\right)-\alpha_{2}\right)^{2}=-4 \frac{1}{\mu \epsilon} \frac{\Delta t^{2}}{\Delta x^{2}} \lambda^{2} A^{2} \alpha_{1}^{2},
$$

where $\alpha_{1}=A^{2}+\gamma\left(1-4 A+6 A^{2}\right)$ and $\alpha_{2}=A^{2}+\gamma\left(4 A^{3}-A^{4}\right)$; which gives the characteristic equation satisfied by the amplification factor:

$$
a_{3} A^{3}+a_{2} A^{2}+a_{1} A+a_{0}=0
$$


with coefficients:

$$
\begin{aligned}
& a_{3}=1+7 \gamma \\
& a_{2}=2(1+6 \gamma)(s \eta \pm j C r \lambda)-8 \gamma \\
& a_{1}=\gamma-1-8 \gamma(s \eta \pm j C r \lambda) \\
& a_{0}=2 \gamma(s \eta \pm j C r \lambda)
\end{aligned}
$$

The sign \pm corresponds to waves propagating in opposite directions and $\mathrm{Cr}=$ $\frac{1}{\sqrt{\mu \epsilon}} \frac{\Delta t}{\Delta x}$ is the Courant number. The characteristic equation has three roots corresponding to one physical mode for each direction and two computational modes ( $2 \Delta t$ and $4 \Delta t$ modes).

Figures $3 \mathrm{a}$ and $3 \mathrm{~b}$ show the magnitude of the amplification factors corresponding to the physical and the computational modes respectively for finite values of $k \Delta x$ obtained from the LF2 scheme using a Courant number of $\mathrm{Cr}=0.3$ and without numerical smoothing $(\nu=0): s=\nu \Delta t / \Delta x^{4}=0$. The value of the filter parameter used for these figures is $\gamma=0.1$. The finite difference approximations of the spatial derivatives are fourth-order accurate and are evaluated on a collocated spatial grid. LF2 produces two unphysical modes corresponding to $2 \Delta t$ and $4 \Delta t$ computational modes (Fig.3b). These modes are both stable. The $4 \Delta t$ mode is strongly damped and vanishes when $k \Delta x=0$ and $k \Delta x=1$. The magnitude of the amplification factors obtained from the unfiltered leapfrog (LF1) and the the standard Robert-Asselin time-filtered leapfrog (LF3) are also superimposed in Figs.3a-b. Both LF1 and LF3 produce one computational mode corresponding to $2 \Delta t$ spurious waves. LF2 and LF3 damp the $2 \Delta t$ computational modes at the same rate of $(1-\gamma) /(1+7 \gamma)$ as $k \Delta x$ approaches 0 because the filter parameters used in these schemes are tuned to satisfy the imposed condition $\gamma_{2}=4 \gamma /(1+7 \gamma)$. The amplification 
factor for the physical mode in LF2 is closer to 1 compared to LF3 indicating that the implicit filtering used in the proposed scheme produces much more accurate solution compared to the filtering commonly used in the standard method (see Fig.1-2). The difference in the amplification factor between LF2 and LF3 becomes more pronounced when the filter coefficient is increased to $\gamma=0.2$ (Fig.3c-d). Figure 3e-h show the magnitude of the amplification factors of the physical and computational modes obtained with a Courant number of $C r=0.5$. The behavior of these modes is similar to that seen in Fig.3a-d with much less damping of the physical mode in LF2 compared to that in LF3. The $2 \Delta t$ computational mode in the unfiltered leapfrog (LF1) is not damped and has an amplification factor with the same magnitude as the physical mode $\left(\left|A_{p}\right|=\left|A_{c}\right|=1\right)$. Although this mode is neutral for linear cases, it is unconditionally unstable for damping terms and may lead to numerical instabilities for nonlinear problems $[20,25]$.

Let us now examine the stability of the LF1, LF2 and LF3 schemes when numerical smoothing is included. Figure 4 shows the magnitude of the amplification factors corresponding to the physical and computational modes for these schemes. Here, we use a numerical smoothing coefficient with a value of $s=\nu \Delta t / \Delta x^{4}=0.004$. This figure shows that both the LF2 and LF3 schemes stabilize and damp the magnitude of the spurious modes, which are well known to be unconditionally unstable for the unfiltered leapfrog scheme (LF1). However, LF3, which uses the classical filtering method degrades the accuracy of the physical modes.

The magnitude of the amplification factors obtained from LF1, LF2 and LF3 using a staggered spatial grid are presented in Fig. 5 with and without numerical smoothing and for a Courant number of $\mathrm{Cr}=0.3$. As for the collocated grid 
(Fig.3-4), they show numerical instabilities for LF1 and conditional stabilities for LF2 and LF3. Again, LF3 produces excessive damping compared to LF2. Thus, LF2 has the advantage of reducing the magnitude of the computational modes while maintaing higher accuracy for the physical modes compared with LF3. This explains the behavior of the numerical solutions presented in Figs.12. The phase errors produced by LF1, LF2 and LF3 are all of second-order. The leapfrog scheme is known to produce acceleration in the phase speed of the numerical solution [26]. The traditional filtering method increases the numerical phase speed compared to the proposed method (Fig. 6). This acceleration is partly compensated or dominated by the deceleration introduced by the finite difference approximations for finite values of $k \Delta x$. Thus, in addition of reducing the computational modes and maintaining higher accuracy for the amplitude of the physical modes, the filtering used in LF2 introduces less phase errors compared to LF3. We note that the staggered grid produces more accurate numerical phase speeds because the finite differencing is more compact and taken over smaller intervals. However, the stability condition for this grid is stricter. The stability criteria for the unfiltered and time-unstaggered leapfrog combined with a collocated spatial grid are $C r<1$ and $C r<0.72$ for second and fourth-order finite differencing respectively. These criteria are $\mathrm{Cr}<0.5$ and $\mathrm{Cr}<3 / 7$ when a staggered spatial grid is used. Thus, the time-unstaggered leapfrog is more stringent that the time-staggered one but it allows explicit treatment of all terms in Maxwell equations by a single scheme including physical damping, numerical smoothing and PML layer contributions. In addition, it does not require solving a constitutive relation for the electric field in nonlinear electromagnetic propagation problems as we will see in the following examples presented in Section 4. 


\section{Application for Maxwell's equations}

\subsection{Example 1: One-dimensional lossy medium}

In this example, we consider a one-dimensional wave propagating in a lossy medium governed by the discretized equations:

$$
\begin{aligned}
& \frac{E_{i}^{n+1}-\bar{E}_{i}^{n-1}}{2 \Delta t}=\frac{1}{\epsilon} \frac{H_{i+1 / 2}^{a v}-H_{i-1 / 2}^{a v}}{\Delta x}-\frac{\sigma}{\epsilon} E_{i}^{n}+\nu\left(\frac{\partial^{4} E}{\partial x^{4}}\right)_{i}^{n} \\
& \frac{H_{p}^{n+1}-\bar{H}_{p}^{n-1}}{2 \Delta t}=\frac{1}{\mu} \frac{E_{p+1 / 2}^{a v}-E_{p-1 / 2}^{a v}}{\Delta x}+\nu\left(\frac{\partial^{4} H}{\partial x^{4}}\right)_{p}^{n}
\end{aligned}
$$

Equations 28 and 29 are integrated by using LF1, LF2 and LF3 schemes within a collocated $(p=i)$ and a staggered $(p=i+1 / 2)$ periodic domains. The expressions of $H^{a v}$ and $E^{a v}$ are given by Eqs.13-14 and Eq.16 for a staggered and collocated grids respectively. The total number of grid points is $N=100$ with a grid spacing of $\Delta x=1.2 \times 10^{-1}$. The values for the filter and the smoothing parameters are $\gamma=0.1$ and $s=\nu \Delta t / \Delta x^{4}=0.004$. The electric conductivity of the medium is $\sigma=1.2 \times 10^{-3}$; and the initial condition for the electric field is $E^{0}(x)=\sin [2 \pi(x-35 \Delta x) / 30 \Delta x]$ for $35 \Delta x \leq x \leq 65 \Delta x$ and $E^{0}(x)=0$, otherwise. Figure 7 shows the numerical solutions obtained from the LF1, LF2, LF3 and RK3 schemes after one revolution around the domain using collocated and staggered grids. The values of the Courant number is $C r=0.3$ for Figs.7a-b and $C r=0.5$ for Fig.7c. As expected, LF1 is unstable and produces spurious oscillations with short wavelengths because of the presence of the current density term (the numerical smoothing is not used in LF1: $s=0$ ). These oscillations are smoothed out in LF2 and LF3. The solution

produced by LF2 is superior and closer to the one computed from RK3. The 
LF3 scheme produces excessive damping of the physical solution compared to LF2.

\subsection{Example 2: Two-dimensional PML layer}

Here, we consider the two-dimensional Maxwell's equations for transversal magnetic waves including a PML absorbing region. The discretized equations solved in this case are:

$$
\begin{aligned}
& \frac{E_{z x(i, j)}^{n+1}-\bar{E}_{z x(i, j)}^{n-1}}{2 \Delta t}=\frac{1}{\epsilon} \frac{H_{y(i+1 / 2, j)}^{a v}-H_{y(i-1 / 2, j)}^{a v}}{\Delta x}-\frac{\sigma}{\epsilon} E_{z x(i, j)}^{n}+\nu\left(\frac{\partial^{4} E_{z x}}{\partial x^{4}}\right)_{(i, j)}^{n} \\
& \frac{E_{z y(i, j)}^{n+1}-\bar{E}_{z y(i, j)}^{n-1}}{2 \Delta t}=-\frac{1}{\epsilon} \frac{H_{x(i, j+1 / 2)}^{a v}-H_{x(i, j-1 / 2)}^{a v}}{\Delta y}-\frac{\sigma}{\epsilon} E_{z y(i, j)}^{n}+\nu\left(\frac{\partial^{4} E_{z y}}{\partial y^{4}}\right)_{(i, j)}^{n} \\
& \frac{H_{y(p, j)}^{n+1}-\bar{H}_{y(p, j)}^{n-1}}{2 \Delta t}=\frac{1}{\mu} \frac{E_{z(p+1 / 2, j)}^{a v}-E_{z(p-1 / 2, j)}^{a v}}{\Delta x}-\frac{\sigma^{*}}{\mu} H_{y(p, j)}^{n}+\nu\left(\frac{\partial^{4} H_{y}}{\partial x^{4}}\right)_{(p, j)}^{n} \\
& \frac{H_{x(i, q)}^{n+1}-\bar{H}_{x(i, q)}^{n-1}}{2 \Delta t}=-\frac{1}{\mu} \frac{E_{z(i, q+1 / 2)}^{a v}-E_{z(i, q-1 / 2)}^{a v}}{\Delta y}-\frac{\sigma^{*}}{\mu} H_{x(i, q)}^{n}+\nu\left(\frac{\partial^{4} H_{x}}{\partial y^{4}}\right)_{(i, q)}^{n}
\end{aligned}
$$

These equations are solved within a collocated $(p=i$ and $q=j)$ and a staggered $(p=i+1 / 2$ and $q=j+1 / 2)$ spatial grids. The two-dimensional domain uses 301 grid points in $x$ and $y$ directions with a grid spacing of $\Delta x=\Delta y=10^{-6}$. The PML absorbing layer uses 10 grid points near the $\mathrm{x}$-and y-boundaries where $\sigma / \epsilon=\sigma^{*} / \mu$ is imposed as a matching condition [14]. The profiles of $\sigma$ and $\sigma^{*}$ use a polynomial function in the form $\sigma(\rho)=\sigma_{\max }(\rho / \delta)^{m}$, where $\delta=10 \Delta x, m=3$ and $\sigma_{\max }$ is calculated such that the reflection coefficient is $10^{-5}$ [14]. The parameters used for the implicit filter and for the numerical smoother are $\gamma=0.2$ and $s=\nu \Delta t / \Delta x^{4}=0.004$ respectively. The initial conditions are given by $E_{z}^{0}(x, y)=\cos \left(\frac{2 \pi r}{L_{1}}\right) \exp \left(-\frac{r^{2}}{L_{2}^{2}}\right)$, where $r=$ $\sqrt{x^{2}+y^{2}}, L_{1}=15 \Delta x$ and $L_{2}=20 \Delta x$. Figure 8 shows the evolution of the 
electric field $E_{z}$ obtained from LF2, LF3 and RK3 on a collocated grid using a Courant number of $\mathrm{Cr}=0.5$. The numerical solutions obtained from LF2 and LF3 are both stable. The waves generated by the initial pulse are absorbed as they enter the PML layer. The solution computed by LF2 is superior compared to that from LF3 and is closer to the reference solution obtained from RK3. Figure 9 shows the numerical results obtained on a staggered grid using a Courant number of $\mathrm{Cr}=0.3$. The waves are effectively absorbed in both cases as they enter the PML region. However, the results using LF2 are much more accurate and less dissipative compared to those using the standard Robert Asselin time-filtered leapfrog scheme (LF3).

The local and global errors due to partial reflection produced as a wave propagates through the PML layer are obtained by conducting a numerical experiment using a benchmark grid that is two times wider than the test grid used in Fig.9. As in [29], the local error within the test grid at the $n$-th time step is defined by $D_{i j}^{\text {local }}=E_{z(i, j)}^{T}-E_{z(i, j)}^{B}$ for all $(i, j)$ within the test grid, where $E_{z}^{T}$ and $E_{z}^{B}$ are the solutions within the test and the benchmark grids respectively. The global reflected error is measured by $D^{\text {global }}=\sum_{i, j}\left(D_{i j}^{l o c a l}\right)^{2}$. Fig.10 shows the local and global reflection errors observed for LF2. The local error (Fig.10a) is calculated at the time step $n=650$ when the peak of the outgoing numerical wave reaches the PML in the test grid (Fig.9c), but is still sufficiently far from the outer boundary of the benchmark grid. The error is normalized by the peak value of the incident wave entering the PML region. It is taken at $j=12$ in the $y$-direction, which is the first row of grid points away from the 10-cell-thick PML used for the test grid. The normalized local reflection is less than $4 \times 10^{-5}$ (Fig.10a), which is very small. The theoretical reflection coefficient is $10^{-5}$. The discrepancy between the numerical and the theoretical reflections has been observed in other studies and was attributed 
to numerical dispersion, phase velocity variations and propagation angle [30].

\subsection{Example 3: Kerr-nonlinear dielectric media}

The performance of the proposed numerical method is also validated by conducting simulations for a nonlinear case where an exact solution can be derived. For this purpose, we consider the Maxwell equations for uniform, thirdorder Kerr-nonlinear dielectric media in one dimension. For this material, the constitutive relation is $D=\left(\epsilon+\chi^{(3)} E^{2}\right) E$, where $\chi^{(3)}$ is the Kerr susceptibility of the material. The time derivative of the displacement and the electric fields are related by $\partial E / \partial t=C(E) \partial D / \partial t$, where $C(E)=\epsilon+3 \chi^{(3)} E^{2}[16]$. The discretized equations solved in this case are:

$$
\begin{aligned}
& \frac{E_{i}^{n+1}-\bar{E}_{i}^{n-1}}{2 \Delta t}=\frac{1}{\epsilon+3 \chi^{(3)} E_{i}^{n 2}} \frac{H_{i+1 / 2}^{a v}-H_{i-1 / 2}^{a v}}{\Delta x}+\nu\left(\frac{\partial^{4} E}{\partial x^{4}}\right)_{i}^{n} \\
& \frac{H_{p}^{n+1}-\bar{H}_{p}^{n-1}}{2 \Delta t}=\frac{1}{\mu} \frac{E_{p+1 / 2}^{a v}-E_{p-1 / 2}^{a v}}{\Delta x}+\nu\left(\frac{\partial^{4} H}{\partial x^{4}}\right)_{p}^{n}
\end{aligned}
$$

The initial conditions consist of a Gaussian pulse $E^{0}(x)=\exp \left[\left(x-x_{o}\right)^{2} / 2 \sigma\right]$

and $H^{0}(x)=\sqrt{\epsilon / \mu} f(\xi) E^{0}(x)$, where $\xi=3 \chi^{(3)} E^{0}(x) / \epsilon, \sigma=5 / \sqrt{2}$ and $x_{o}=15$. The parameters of the material are $\epsilon=1, \mu=1$ and $\chi^{(3)}=-0.08$. The function $f(\xi)$ and the analytical solution within the time interval $[0,15]$ for this case are given in [16]. The computational domain uses 200 grid points within collocated and staggered grids with a grid spacing $\Delta x=0.25$. The parameters of the simulations are $\gamma=0.1, s=\nu \Delta t / \Delta x^{4}=0.004$; with Courant number values of $C r=0.5$ and $C r=0.3$ for the collocated and the staggered grids respectively. We note that the scheme presented in Eqs.30-31 does not require solving the constitutive relation for the electric field at each time step 
as in the standard FDTD methods. Fig.11 superimposes the exact electric field profile at $t=15$ and its numerical approximations using the LF2 scheme within a collocated and a staggered grid. As shown in this figure, the initial pulse steepens as it propagates from left to right in the nonlinear medium. The numerical and the exact solutions are indistinguishable from each other demonstrating that the proposed scheme can be applied to nonlinear electromagnetic wave propagation problems.

\subsection{Example 4: Application to parametric four wave mixing in an optical nonlinear Kerr medium}

In this example, we use the quantitative verification technique proposed in [31] to test our computational method. In this rigorous verification technique, parametric Four-Wave Mixing (FWM) in an optical Kerr medium is analyzed and compared against analytical asymptotic solutions under realistic conditions. The technique has been employed to investigate the accuracy of nonlinear FDTD algorithms in modeling optical pulse propagation in a nonlinear medium [32].

FWM is a nonlinear phenomenon caused by the third-order optical nonlinearity. The optical parametric process of FWM occurs when a strong pump wave with an electric field amplitude $E_{p}$ and frequency $\omega_{p}$ is propagating together with a weak signal wave with an amplitude $E_{s}$ and frequency $\omega_{s}>\omega_{p}$ in an optical medium characterized by the third-order instantaneous Kerr nonlinearity that is represented by the nonlinear electric susceptibility $\chi^{(3)}$. In this process, two additional converted waves with the frequencies $\omega_{1}=2 \omega_{p}-\omega_{s}$ and $\omega_{2}=2 \omega_{s}-\omega_{p}$ are produced by a nonlinear refractive index modulation of the medium $[31,32]$. If the pump wave is sufficiently larger than the signal 
wave $\left(E_{p}>>E_{s}\right)$, and the signal wave is sufficiently larger than the converted wave, an analytical asymptotic solution expressing the small signal FWM conversion efficiency $\eta_{s}$ for a plane wave at the frequency $2 \omega_{p}-\omega_{s}$ can be derived $[31,32]$. It is given by the following relation:

$$
\eta_{s}=\left(\frac{3 \omega_{p} \chi^{(3)}}{8 n c} E_{p}^{2} L\right)^{2}
$$

where $E_{p}$ is the electric field amplitude of the pump wave, $L$ is the propagation distance, $n=\sqrt{\epsilon_{r}}$ is the linear refractive index of the entire medium and $c$ is the speed of light. The third-order nonlinear susceptibility is chosen as a typical value of nonlinear optical materials such as InGaAsP, that is $\chi^{(3)}=1.0 \times 10^{-18}$ $\mathrm{m}^{2} / \mathrm{V}^{2}[31,33,34]$.

The two-dimensional discretized equations that describe the evolution of field components $E_{z}, H_{x}$ and $H_{y}$ in the presence of FWM phenomenon are:

$$
\begin{aligned}
\frac{E_{z x(i, j)}^{n+1}-\bar{E}_{z x(i, j)}^{n-1}}{2 \Delta t} & =\frac{1}{\epsilon_{0}\left(\epsilon_{r}+3 \chi^{(3)}\left|\mathbf{E}_{(i, j)}^{n}\right|^{2}\right)} \frac{H_{y(i+1 / 2, j)}^{a v}-H_{y(i-1 / 2, j)}^{a v}}{\Delta x} \\
& -\frac{\sigma}{\epsilon} \frac{E_{z x(i, j)}^{n+1}+\bar{E}_{z x(i, j)}^{n-1}}{2}+\nu\left(\frac{\partial^{4} E_{z x}}{\partial x^{4}}\right)_{(i, j)}^{n} \\
\frac{E_{z y(i, j)}^{n+1}-\bar{E}_{z y(i, j)}^{n-1}}{2 \Delta t} & =-\frac{1}{\epsilon_{0}\left(\epsilon_{r}+3 \chi^{(3)}\left|\mathbf{E}_{(i, j)}^{n}\right|^{2}\right)} \frac{H_{x(i, j+1 / 2)}^{a v}-H_{x(i, j-1 / 2)}^{a v}}{\Delta y} \\
& -\frac{\sigma}{\epsilon} \frac{E_{z y(i, j)}^{n+1}+\bar{E}_{z y(i, j)}^{n-1}}{2}+\nu\left(\frac{\partial^{4} E_{z y}}{\partial y^{4}}\right)_{(i, j)}^{n} \\
\frac{H_{y(p, j)}^{n+1}-\bar{H}_{y(p, j)}^{n-1}}{2 \Delta t} & =\frac{1}{\mu_{0}} \frac{E_{z(p+1 / 2, j)}^{a v}-E_{z(p-1 / 2, j)}^{a v}}{\Delta x} \\
& -\frac{\sigma^{*}}{\mu_{0}} \frac{H_{y(p, j)}^{n+1}+\bar{H}_{y(p, j)}^{n-1}}{2}+\nu\left(\frac{\partial^{4} H_{y}}{\partial x^{4}}\right)_{(p, j)}^{n} \\
\frac{H_{x(i, q)}^{n+1}-\bar{H}_{x(i, q)}^{n-1}}{2 \Delta t} & =-\frac{1}{\mu_{0}} \frac{E_{z(i, q+1 / 2)}^{a v}-E_{z(i, q-1 / 2)}^{a v}}{\Delta y}
\end{aligned}
$$




$$
-\frac{\sigma^{*}}{\mu_{0}} \frac{H_{x(i, q)}^{n+1}+\bar{H}_{x(i, q)}^{n-1}}{2}+\nu\left(\frac{\partial^{4} H_{x}}{\partial y^{4}}\right)_{(i, q)}^{n}
$$

where $\epsilon=\epsilon_{0} \epsilon_{r}$ is the dielectric permittivity of the optical medium with refractive index $n=\sqrt{\epsilon_{r}}=1.2 ; \epsilon_{0}$ and $\mu_{0}$ are the permittivity and the magnetic permeability of vacuum and the nonlinear electric susceptibility is $\chi^{(3)}=1.0 \times 10^{-18} \mathrm{~m}^{2} / \mathrm{V}^{2}$. These values are the same as the one used in [31]. Note that we have introduced a semi-implicit treatment for the PML terms in Eqs. 33-36 to demonstrate that the proposed method can also be employed in combination with a semi-implicit scheme. Although the scheme utilizes an implicit time filter in that both $E_{z x}^{n+1}\left(E_{z y}^{n+1}, H_{x}^{n+1}, H_{y}^{n+1}\right)$ and $\bar{E}_{z x}^{n-1}\left(\bar{E}_{z y}^{n-1}\right.$, $\bar{H}_{x}^{n-1}, \bar{H}_{y}^{n-1}$ ) are not known as they depend on each other (Eqs. 5-6), the semi-implicit treatment of the PML terms can be formulated explicitly. The formulation for the electric field component $E_{z x}^{n+1}$ is as follows:

$$
E_{z x}^{n+1}=\frac{1}{\beta}\left[(1-\sigma \Delta t / \epsilon) \tilde{E}_{z x}^{n-1}+(1+6 \gamma) 2 \Delta t F^{n}\right]
$$

where $\beta=(1+7 \gamma)+(1+5 \gamma) \sigma \Delta t / \epsilon, F^{n}$ represents the explicit terms in Eq. 33 (the first and third terms in the right hand side) and $\tilde{E}_{z x}^{n-1}$ is given by Eq.9 after replacing $\mathbf{D}$ by $E_{z x}$. The two-dimensional numerical solutions obtained by this scheme use grid spacing of $\Delta x=\Delta y=0.01 \mu \mathrm{m}$ and spatial discretization with fourth-order finite difference approximations formulated in flux forms given by Eq. 13-14. Note that 2-D verifications of FWM were not performed in $[31,32]$. We consider a dielectric medium with a total length of $L=12 \mu \mathrm{m}$. The structure consists of a dielectric slab for $-0.7 \mu \mathrm{m}<\mathrm{y}<$ $0.7 \mu \mathrm{m}$, with permittivity value $\epsilon=12.7310^{-12} \mathrm{~F} \mathrm{~m}^{-1}$ located at $x<1 \mu \mathrm{m}$ and a free space for $|y|>0.7 \mu \mathrm{m}$. The width of the structure is $d=4 \mu \mathrm{m}$ for $x>1 \mu \mathrm{m}$ (see Fig. 13). In addition, the structure is separated into a linear 
medium for $x<2 \mu \mathrm{m}$ where $\chi^{(3)}=0$ and a nonlinear material for $x>2 \mu \mathrm{m}$ where the nonlinear susceptibility is given by $\chi^{(3)}=1.0 \times 10^{-18} \mathrm{~m}^{2} / \mathrm{V}^{2}$. The simulation utilizes a pump wave with an amplitude of $E_{p}=5.1 \times 10^{7} \mathrm{~V} / \mathrm{m}$ and a frequency of $192 \mathrm{THz}$ supplemented by a signal wave with an amplitude which is $20 \mathrm{~dB}$ lower than the pump wave and a frequency of $195 \mathrm{THz}$. The FDTD calculations were conducted for the time period of 4 ps. Time signals are then collected at the right exit after propagation through the nonlinear medium. The intensity of the converted wave is then calculated by performing spectral analysis where time series were tapered with a Kaizer window to suppress spectral leakage and reduce the side lobes in the spectrum, enabling resolution of a converted signal with an intensity as small as $60 \mathrm{~dB}$ lower than the intensity of the pump wave. The spectra presented in Fig. 12a are normalized by the maximum value so that the power spectrum at the pump wave frequency $(192 \mathrm{THz})$ is $0 \mathrm{~dB}$. We recall that the signal wave uses an amplitude value of $20 \mathrm{~dB}$ lower than the pump wave and a specified frequency of $195 \mathrm{THz}$. According to the FWM theory, the converted waves are predicted at $189 \mathrm{THz}$ and $198 \mathrm{THz}$. These converted waves are clearly evident in the spectra shown in Fig. 12a. Other converted waves with smaller amplitudes are also observed around $187 \mathrm{THz}$ and $202 \mathrm{THz}$ in the power spectra with peak values that are lower than $-100 \mathrm{~dB}$ suggesting other nonlinearly generated signal waves. Linear simulations using the same parameters as in Fig. 12a except that $\chi^{(3)}=0$ were conducted. The resulting spectra are shown in Fig 12b. The two maxima seen in this figure correspond to the imposed pump and signal waves. It is obvious that the FWM wave conversion mechanism is not observed in this case. Thus, the converted waves are suppressed when nonlinearity is removed. The value of the power spectrum of the converted signal observed for the 2-D cylindrical waves at $189 \mathrm{THz}$ is smaller than the 
value obtained for a plane (1D) wave, which is also superimposed in Fig 12a. The plane wave produces a value of $-49.89 \mathrm{~dB}$. The corresponding conversion efficiency relative to the signal wave is $-29.89 \mathrm{~dB}$, which is very close to the theoretical value of the conversion efficiency for a plane wave obtained from Eq. $32\left(\eta_{s}=-29.71 \mathrm{~dB}\right)$. Fig.13a shows the electric field profile simulated within the nonlinear structure. The waves are excited within the dielectric slab. The guided waves propagate from left to right through the nonlinear dielectric medium and are absorbed by PML layers surrounding the material. Fig.13b shows the difference in the electric field between the nonlinear and the linear simulations. The difference between these fields is observed only in the nonlinear region $(x>2 \mu \mathrm{m})$ where FWM is acting and producing converted waves by nonlinear refractive index modulation of the medium. Figs. 13ab do not show noticeable wave reflection at the boundaries of the medium demonstrating the effectiveness of the formulation and implementation of the PML terms in Eqs.33-36.

\section{$5 \quad$ Summary}

In this paper, we have presented a numerical method for integration of the time dependent Maxwell equations. This method uses temporal unstaggered grids and is based on the leapfrog scheme with a fourth-order implicit time filter. It employs fourth-order accurate finite difference approximations within collocated and staggered grids. We showed that the proposed computational scheme allows explicit treatment of physical and artificial damping, namely terms involving electric current density and selective numerical smoothing, which can be introduced in Maxwell equations to smooth out short oscillations inherent when finite differencing is used to approximate spatial derivatives. 
Comparison of simulation results showed that the proposed method is robust and effective in smoothing out oscillations generated by spatial differencing. It produces numerical approximations that are more accurate than those computed by the unfiltered leapfrog scheme and the classical Robert-Asselin time filtered method.

Stability analysis and examples of application to Maxwell equations demonstrated that the proposed scheme has the ability to maintain higher accuracy for the physical modes while stabilizing and controlling numerical instabilities of computational modes in electromagnetic wave propagation problems including fourth-order selective numerical smoothing. The computational modes become unconditionally unstable when the unfiltered leapfrog is applied. The classical filtering produces excessive dissipation and degrades the accuracy of the physical solution. These examples also demonstrated that the method is robust and effective when employed within PML regions. It does not require solving a constitutive relation for the electric field in nonlinear electromagnetic propagation problems. The performance of the computational algorithm is also verified by analyzing parametric four wave mixing in an optical nonlinear Kerr medium. In our future work, we will apply the numerical method to solutions of three-dimensional stochastic Maxwell and Navier-Stokes Equations ([35]).

\section{Acknowledgments}

We acknowledge support from the NSF DMS grant 1419593. The authors are supported in part by the Wilhoit Foundation at ASU, AFOSR and NSF Earth System Models (EaSM) program. 


\section{References}

[1] K. S. Yee, 1966: Numerical solution of initial boundary values problems involving Maxwell's equations in isotopic media. IEEE Tran. Antennas Propagat., 14(3), 302 - 307.

[2] A. Taflove, S. G. Johnson and A. Oskooi, 2013: Advances in FDTD Computational Electrodynamics: Photonics and Nanotechnology. Artech House, Boston, MA.

[3] T. G. Jurgens, A. Taflove, K. Umashankar, and T. G. Moore, 1992: Finitedifference time-domain modeling of curved surfaces, IEEE Trans. Antennas Propag. 40, 357 - 366.

[4] W. H. Yu and R. Mittra, 2000: A conformal FDTD algorithm for modeling perfectly conducting objects with curve-shaped surfaces and edges, Microw. Opt. Technol. Lett. 27, 136 - 138.

[5] A. Yefet and P. G. Petropoulos, 2001: A staggered fourth-order accurate explicit finite difference scheme for the time-domain Maxwell's equations, J. Comput. Phys. 168, $286-315$.

[6] Y. Liu, 1996: Fourier analysis of numerical algorithms for the Maxwell's equations, J. Comput. Phys. 124, 396 - 416.

[7] S. V. Georgakopoulos, C. R. Birtcher, C. A. Balanis, and R. A. Renaut, 2002: Higher- order finite-difference schemes for electromagnetic radiation, scattering, and penetration, Part I: Theory, IEEE Antennas Propag. Mag. 44, 134 - 142.

[8] J. Fang, 1989: Time domain finite difference computation for Maxwell's equations, Ph.D. dissertation, Univ. California, Berkeley, CA.

[9] Z. Bi, K.Wu, C.Wu, and J. Litva, 1991: A new finite-difference time-domain algorithm for solving Maxwell's equations, IEEE Microwave Guided Wave Lett., $1,382-384$. 
[10] Z. Chen, M. M. Ney, and W. J. R. Hoefer, 1991: A new finite-difference timedomain formulation and its equivalence with the TLM symmetrical condensed node, IEEE Trans. Microwave Theory Tech., 39, 2160 - 2169.

[11] K. L. Shlager, J. G. Maloney, S. L. Ray, and A. F. Peterson, 1993: Relative accuracy of several finite-difference time-domain methods in two and three dimensions, IEEE Trans. Antennas Propagat., 41, 1732 - 1737.

[12] G. Sun and C.W. Trueman, 2005: Optimized finite difference time domain methods based on the $(2,4)$ stencil, IEEE Trans. Microw. Theory Tech. 53, $832-842$.

[13] K. L Shlager and J. B. Schneider, 2003: Comparison of the Dispersion Properties of Several Low-Dispersion Finite-Difference Time-Domain Algorithms, IEEE Trans. Antennas Propagat., vol. 51, 642 - 653.

[14] J. P. A. Berenger, 1994: Perfectly matched layer for the absorption of electromagnetic waves, J. Comput. Phys. 114, 185-200.

[15] J. P. A. Berenger, 2007: Perfectly Matched Layer (PML) for Computational Electromagnetics. Morgan and Claypool Publishers series.

[16] M. Pototschnig, M., J. Niegemann, L. Tkeshelashvili, and K. Busch, 2009: Time-domain simulations of the nonlinear Maxwell equations using operatorexponential methods, IEEE Trans. Antennas Propag., 57, 475-483.

[17] B. Finkelstein and R. Kastner, 2007: Finite difference time domain dispersion reduction schemes, Journal of Computational Physics, 221, 422 - 438.

[18] J. L. Young, D. Gaitonde, and J. S. Shang, 1997: Toward the construction of a fourth- order difference scheme for transient EM wave simulation: Staggered grid approach, IEEE Trans. Antennas Propag. 45, 1573 - 1580.

[19] J. S. Shang, 1999: High-order compact-difference schemes for time-dependent Maxwell equations, J. Comput. Phys. 153, 312 - 333. 
[20] M. Moustaoui, A. Mahalov and E. J. Kostelich, 2014: A numerical method based on Leapfrog and a fourth-order implicit time filter, Mon. Wea. Rev., 142, $2545-2560$.

[21] A. J., Robert, 1966: The integration of a low order spectral form of the primitive meteorological equations. J. Meteor. Soc. Japan, 44, 237 - 245.

[22] R. Asselin, 1972: Frequency filter for time integrations. Mon. Wea. Rev, 100, $487-490$.

[23] W. C. Skamarock and J. B. Klemp, 2008: A time-split nonhydrostatic atmospheric model for weather research and forecasting applications. J. of Comp. Phys., 227, $3465-3485$.

[24] L. J. Wicker and W. C. Skamarock, 2002: Time splitting methods for elastic models using forward time schemes. Mon. Wea. Rev., 130, 2088 - 2097.

[25] D. R. Durran, 1991: The third-order Adams-Bashforth method: An attractive alternative to leapfrog time diferencing. Mon. Wea. Rev., 119, 702 - 720.

[26] D. R., Durran, 1999: Numerical Methods for Wave Equations in Geophysical Fluid Dynamics. Springer-Verlag, 482 pp.

[27] A. Mahalov and M. Moustaoui, 2009: Vertically Nested Nonhydrostatic Model for Multi-Scale Resolution of Flows in the Upper Troposphere and Lower Stratosphere, Journal of Computational Physics, 228, 1294 - 1311.

[28] P. K. Smolarkiewicz and J. Szmelter, 2009: Iterated upwind schemes for gas dynamics. J. Comput. Phys., 228, 33-54.

[29] T. G. Moore, J. G. Blaschak, A. Taflove, and G. A. Kriegsmann, 1988: Theory and application of radiation boundary operators. IEEE Trans. Antennas Propag., 36, 1797-1812.

[30] A. Taflove, 1995: Computational Electrodynamics: The Finite-Difference TimeDomain Method. Norwood, MA, Artech House, 595 pp. 
[31] M. Fujii, C. Koos, C. Poulton, I. Sakagami, J. Leuthold, and W. Freude: 2006, A simple and rigorous verification technique for nonlinear FDTD algorithms by optical parametric four-wave mixing, Microw. Opt. Technol. Lett., 48, 88 - 91.

[32] I. S. Maksymov, A. A. Sukhorukov, A. V. Lavrinenko and Y. S. Kivshar: 2011, Comparative study of FDTD-adopted numerical algorithms for Kerr nonlinearities, IEEE Antennas and Wireless Propagat. Lett., 10, 143 - 146.

[33] Y. Xiang, X. Zhang, W. Cai, L. Wang, C. Ying and J. Xu: 2013, Optical bistability based on Bragg grating resonators in metal-insulator-metal plasmonic waveguides, AIP Advances 3, 012106.

[34] R. C. Shiu,Y. C. Lan and G. Y. Guo: 2014, Optical multiple bistability in metal-insulator-metal plasmonic waveguides side-coupled with twin racetrack resonators, J. Opt. Soc. Am. B, 31, 2581-2586.

[35] F. Flandoli and A. Mahalov, 2012: Stochastic 3D rotating Navier-Stokes equations: averaging, convergence and regularity, Archive for Rational Mechanics and Analysis, 205, 195 - 237. 

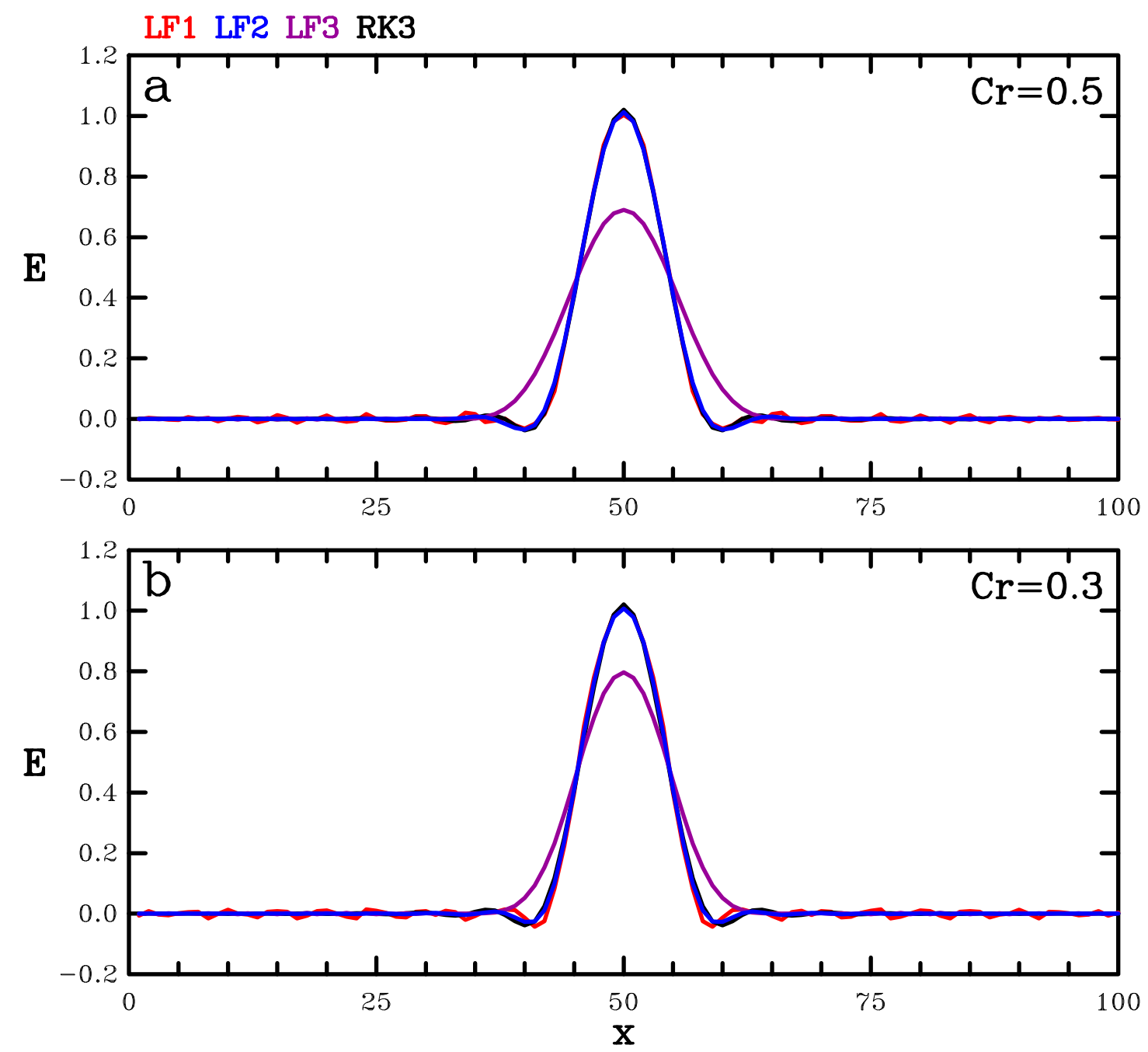

Fig. 1. Comparison of numerical solutions to Eqs.11-12 obtained from the unfiltered leapfrog scheme (LF1, red), the proposed method (LF2, blue), the standard Robert-Asselin time filtered scheme (LF3, purple) and RK3 (black). The spatial differencing uses fourth-order accurate approximations evaluated on a collocated grid. The Courant numbers are (a) $C r=0.5$ and (b) $C r=0.3$. Numerical smoothing is not applied to LF1. LF2, LF3 and RK3 use a smoothing coefficient of $s=\nu \Delta t / \Delta x^{4}=0.004$. 


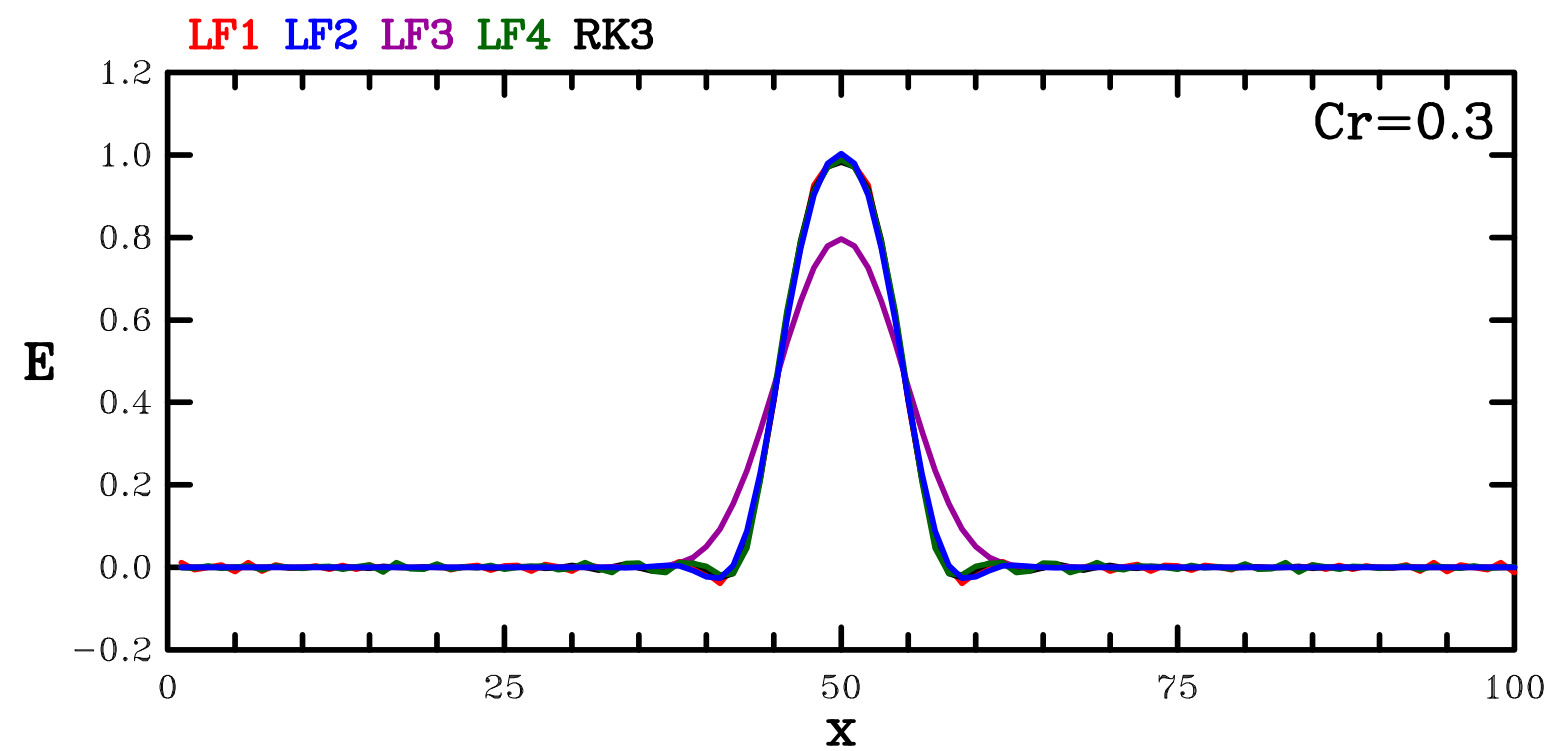

Fig. 2. Comparison of numerical solutions to Eqs.11-12 obtained from the unfiltered leapfrog scheme (LF1, red), the proposed method (LF2, blue), the standard Robert-Asselin time filtered scheme (LF3, purple), the time-staggered leapfrog scheme (LF4, green) and RK3 (black). The spatial differencing uses fourth-order accurate approximations evaluated on a staggered grid. Courant number is $\mathrm{Cr}=0.3$. Numerical smoothing is not applied to LF1 and LF4. LF2, LF3 and RK3 use a smoothing coefficient of $s=\nu \Delta t / \Delta x^{4}=0.004$. 


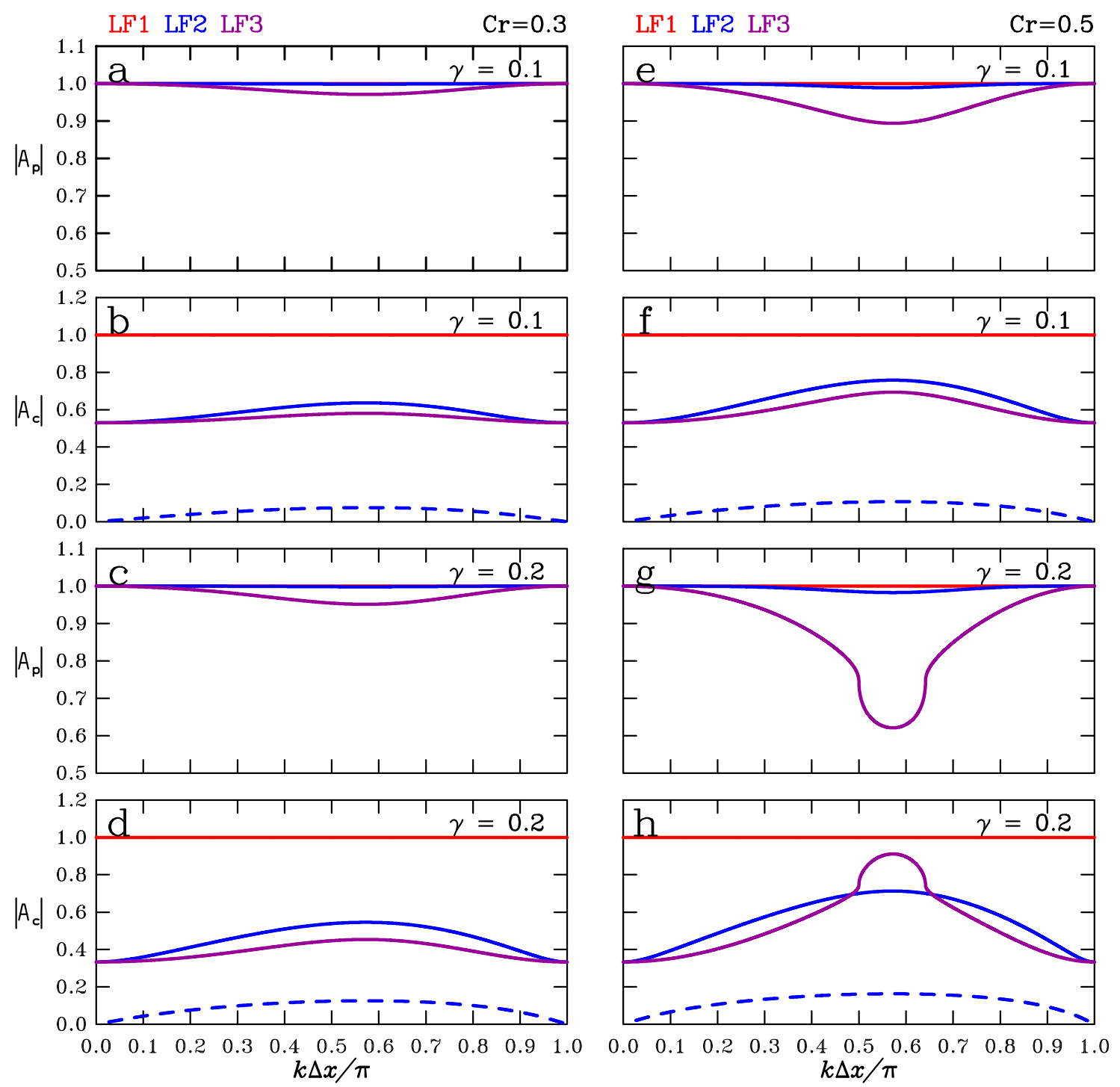

Fig. 3. Magnitudes of the amplification factors for the physical mode (a) and the computational modes (b) obtained from the unfiltered leapfrog scheme (LF1), the proposed method (LF2) and the standard Robert-Asselin time filtered scheme (LF3) with a filter coefficient $\gamma=0.1$ and Courant number $\mathrm{Cr}=0.3$. (c) and (d) are the same as (a) and (b) respectively, but with $\gamma=0.2$. The spatial differencing uses fourth-order accurate approximations evaluated on a collocated grid without numerical smoothing $\left(s=\nu \Delta t / \Delta x^{4}=0\right)$. (e), (f), (g) and (h) are the same as (a), (b), (c), and (d) but for $C r=0.5$. 

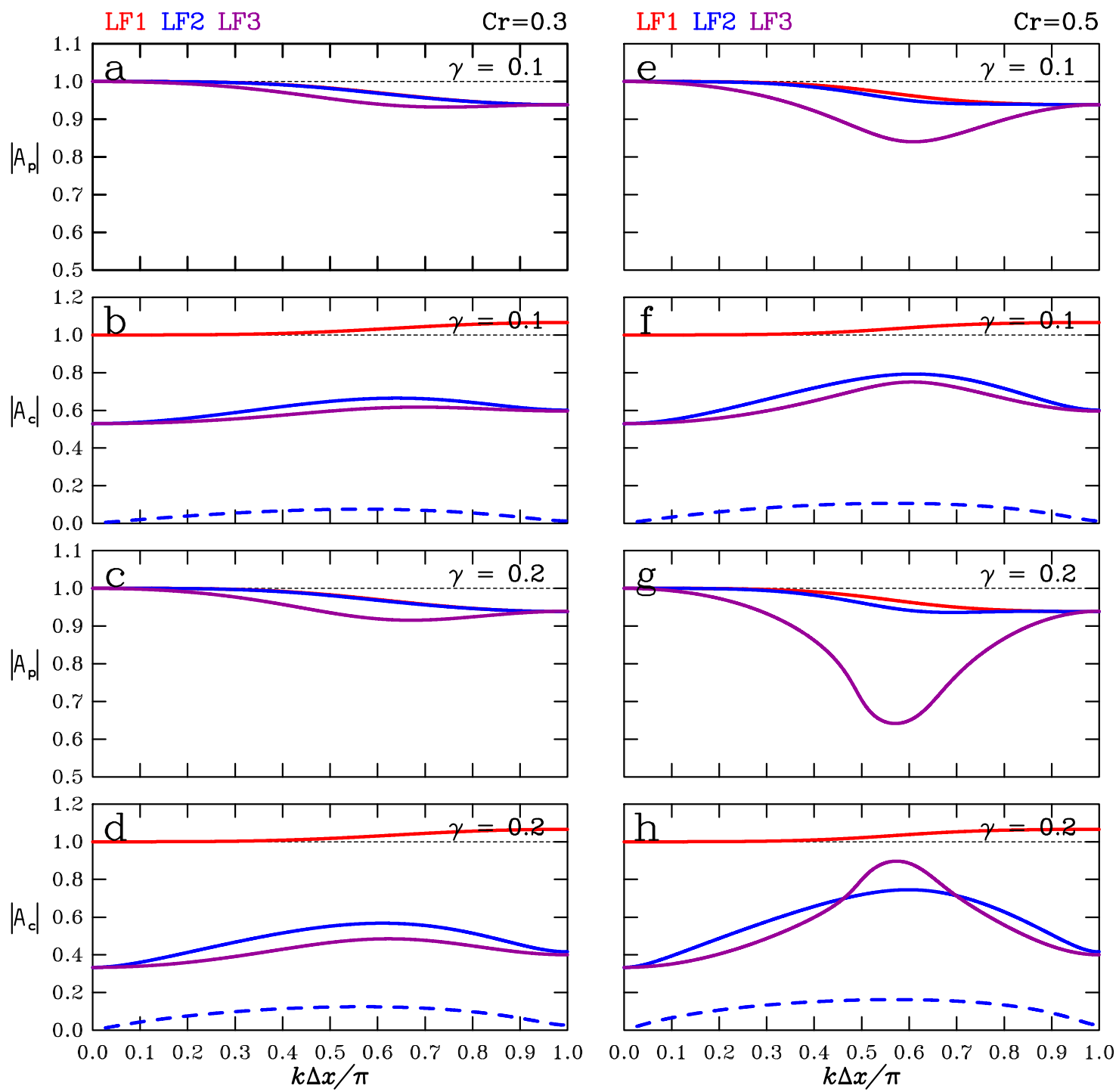

Fig. 4. Same as Fig.3 but with numerical smoothing $\left(s=\nu \Delta t / \Delta x^{4}=0.004\right)$. 

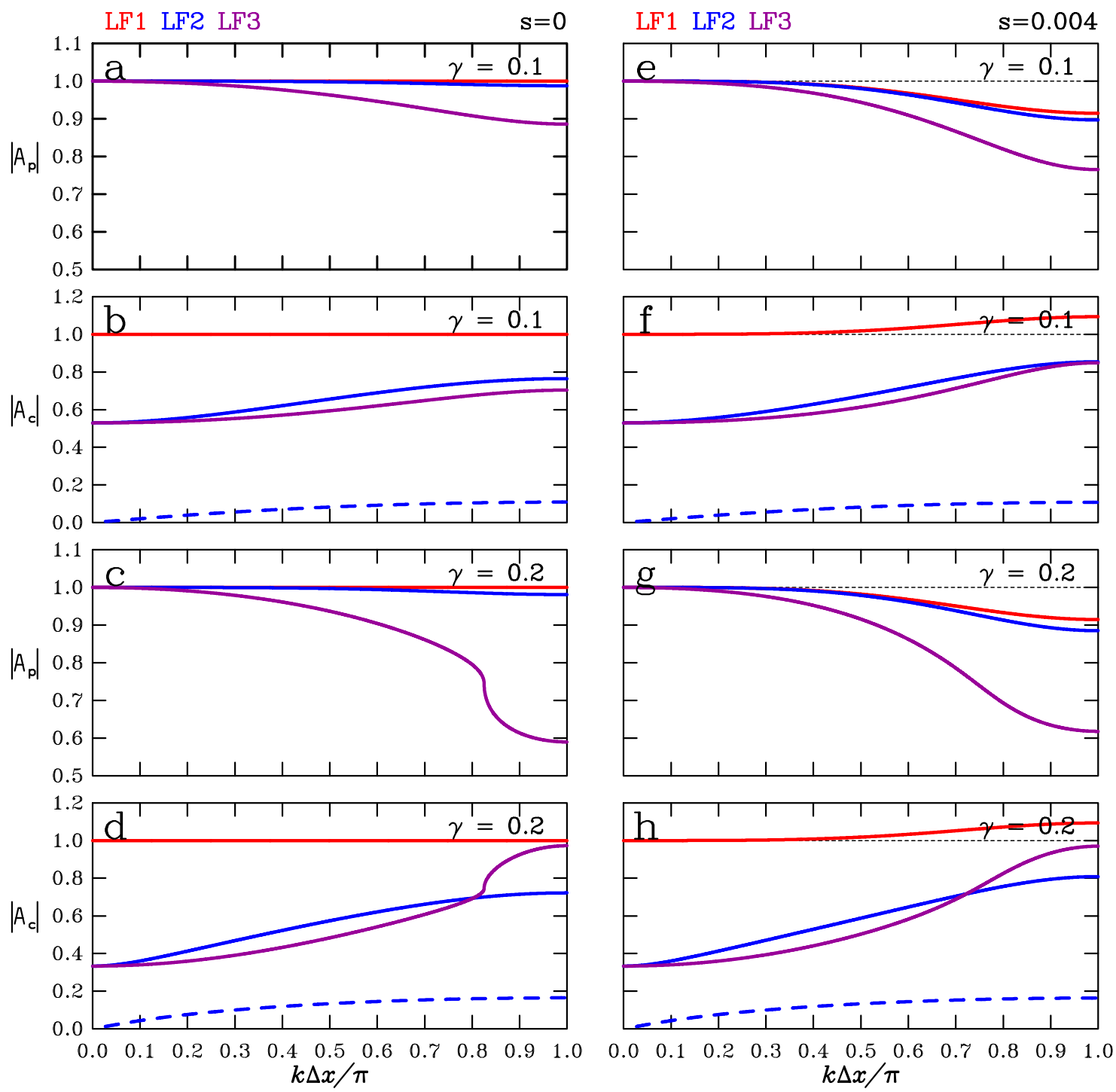

Fig. 5. Magnitudes of the amplification factors for the physical mode (a) and the computational modes (b) obtained from the unfiltered leapfrog scheme (LF1), the proposed method (LF2) and the standard Robert-Asselin time filtered scheme (LF3) with a filter coefficient $\gamma=0.1$ and without numerical smoothing. (c) and (d) are the same as (a) and (b) respectively, but with $\gamma=0.2$. The spatial differencing uses fourth-order accurate approximations evaluated on a staggered grid with Courant number $C r=0.3$. (e), (f), (g) and (h) are the same as (a), (b), (c), and (d) but with numerical smoothing $\left(s=\nu \Delta t / \Delta x^{4}=0.004\right)$. 

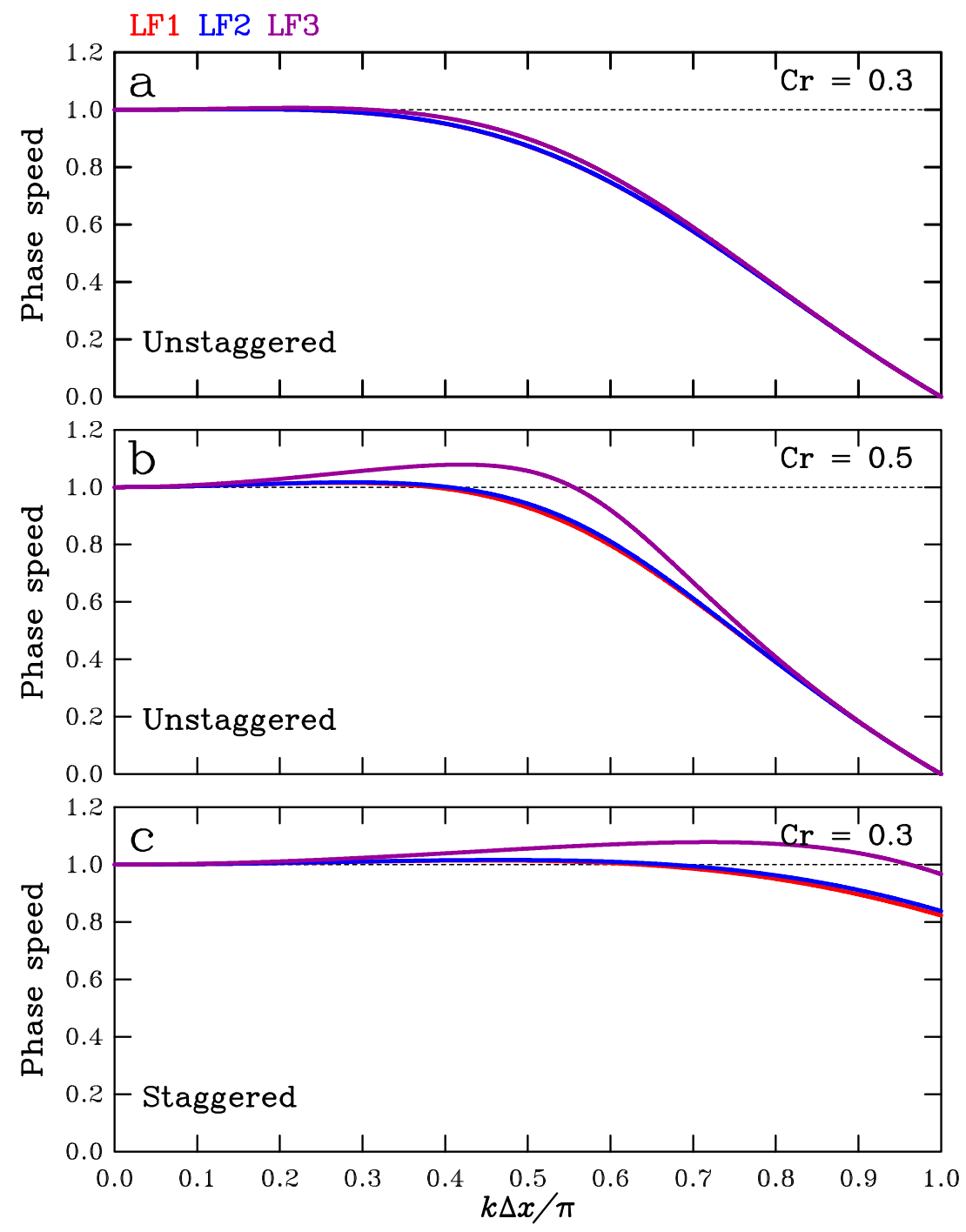

Fig. 6. Relative phase speed for the physical mode obtained from the unfiltered leapfrog scheme (LF1), the proposed method (LF2) and the standard Robert-Asselin time filtered scheme (LF3) with a filter coefficient $\gamma=0.1$. The spatial differencing uses fourth-order accurate approximations evaluated on a collocated grid with Courant numbers (a): $C r=0.3$ and (b): $C r=0.5$. (c) is the same as (a) but the grid is staggered. 

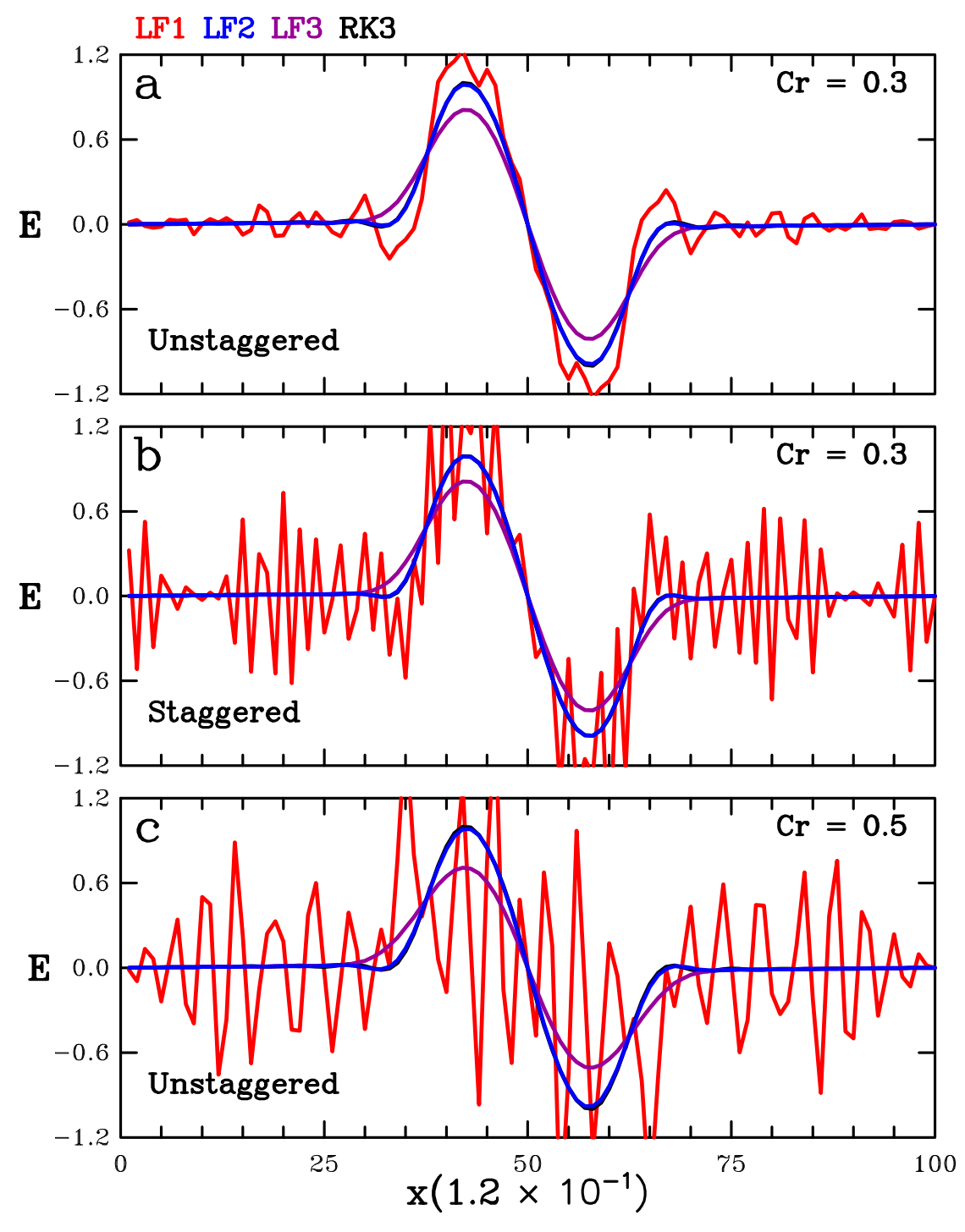

Fig. 7. Comparison of numerical solutions to Eqs.28-29 for the electric field in lossy medium, with electric conductivity $\sigma=1.2 \times 10^{-3}$, obtained from the unfiltered leapfrog scheme (LF1), the proposed method (LF2), the standard Robert-Asselin time filtered scheme (LF3) and RK3. The spatial differencing uses fourth-order accurate approximations evaluated on (a): collocated and (b): staggered grids with Courant number $C r=0.3$. (c) is the same as (a) but with $C r=0.5$. Numerical smoothing is not applied to LF1. LF2, LF3 and RK3 use a smoothing coefficient of $s=\nu \Delta t / \Delta x^{4}=0.004$. 


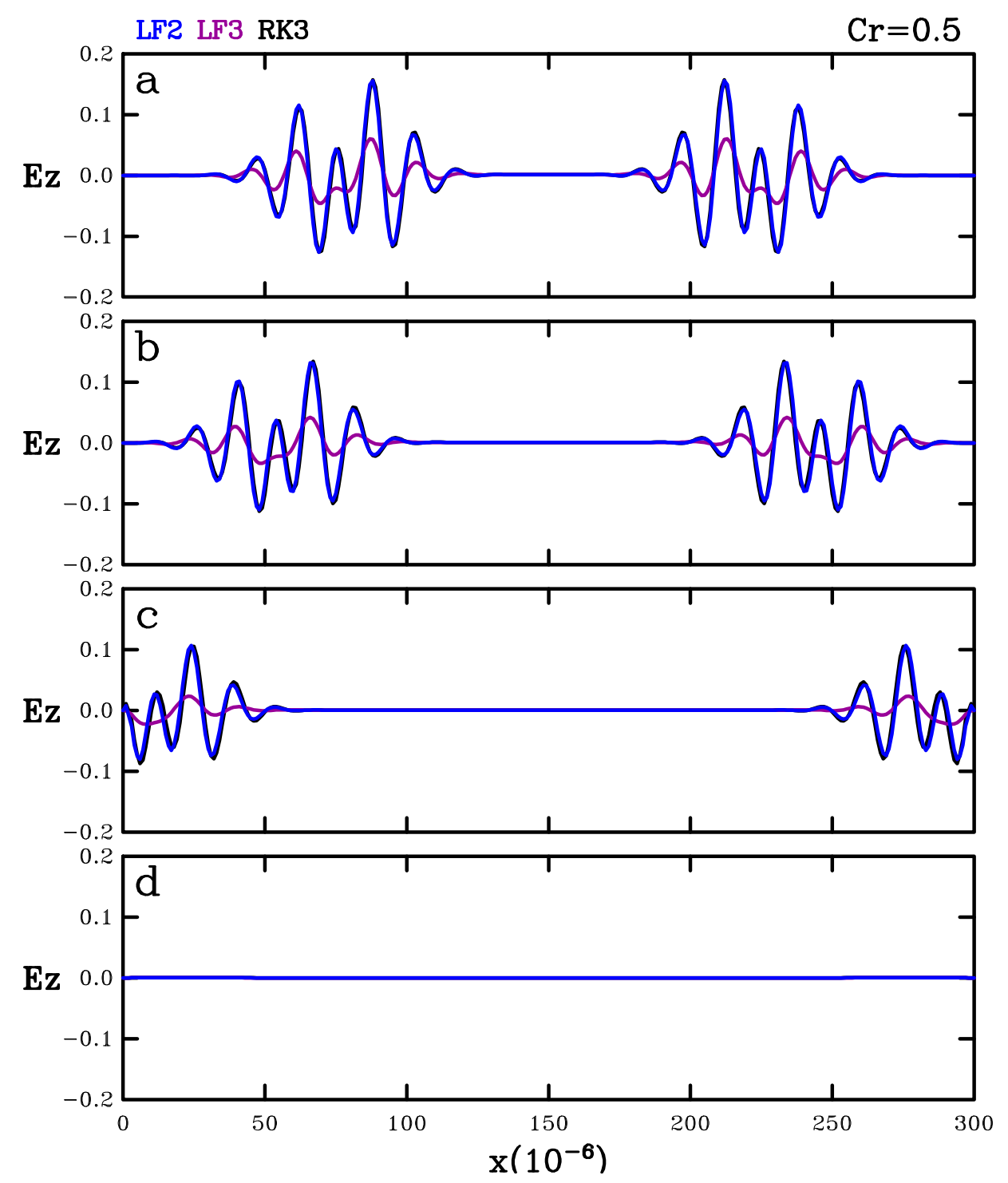

Fig. 8. Sequence of electric field profiles illustrating the absorption of an electromagnetic wave propagating through a PML layer (see text). The profiles are computed from the proposed method (LF2), the standard Robert-Asselin time filtered scheme (LF3) and RK3 after (a): 210, (b): 270, (c): 390 and (d): 570 time steps. The spatial differencing uses fourth-order accurate approximations evaluated on a collocated grid with Courant number $\mathrm{Cr}=0.5$. The smoothing coefficient is $s=\nu \Delta t / \Delta x^{4}=0.004$. 


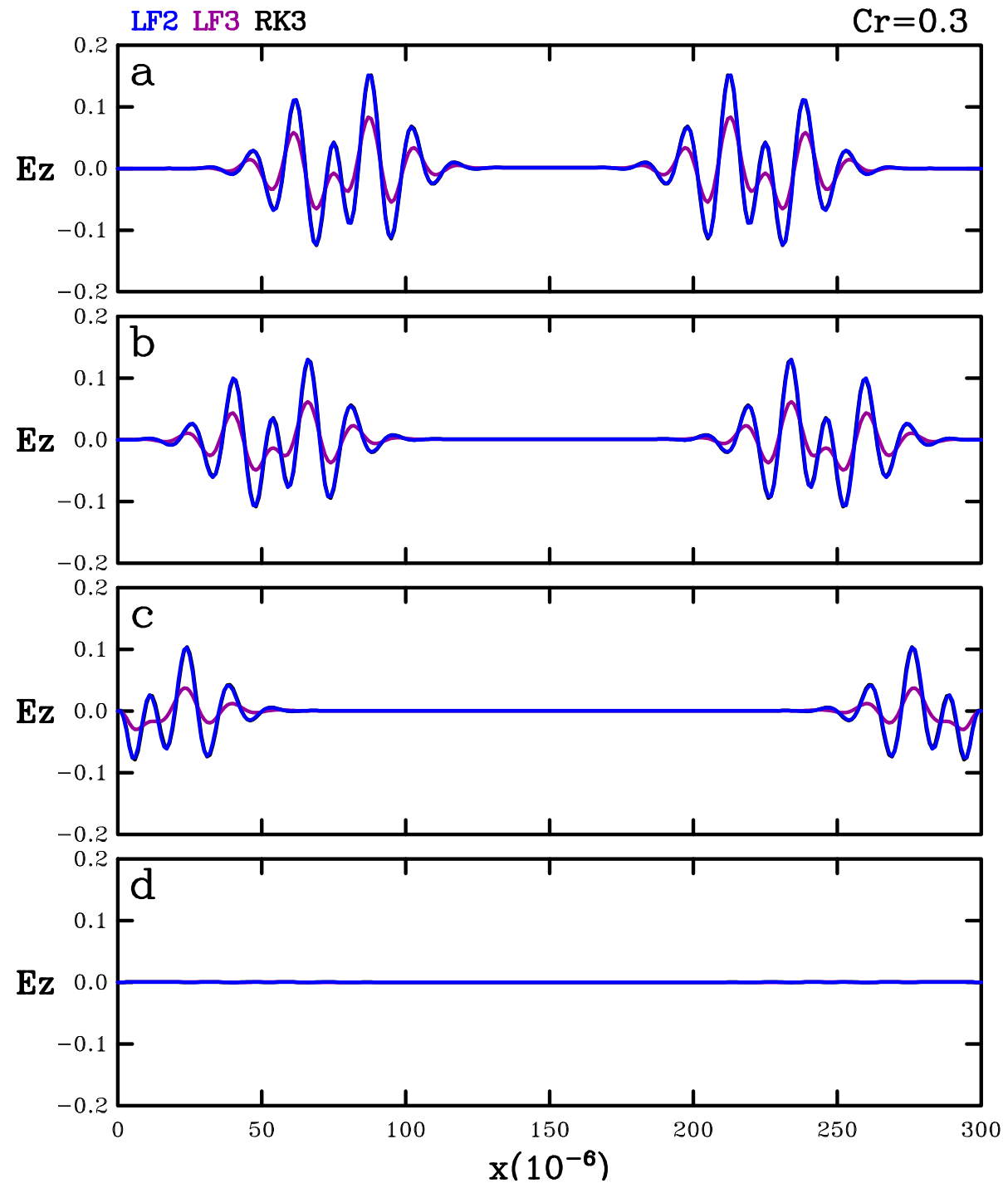

Fig. 9. Same as Fig.8 but the grid is staggered, the Courant number is $\mathrm{Cr}=0.3$, and the profiles are computed after (a): 350, (b): 450, (c): 650 and (d): 950 time steps. 

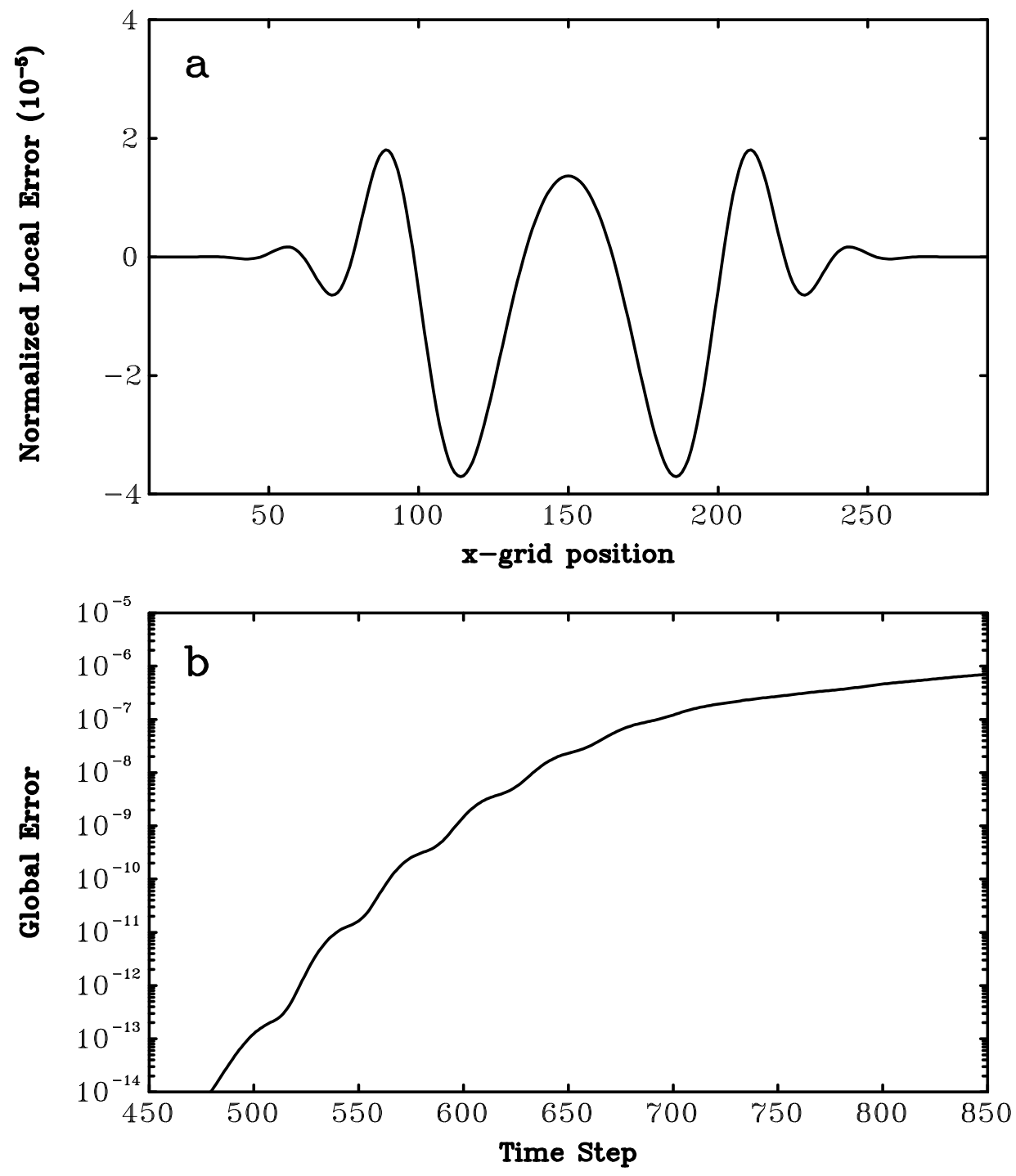

Fig. 10. Error measures computed by the LF2 scheme from the numerical simulations presented in Fig.9: (a) normalized local error at the time step $n=650$ along the first row of grid points away from the PML layer in the y-direction $(j=12)$; (b) global error as a function of time step within the test grid. 


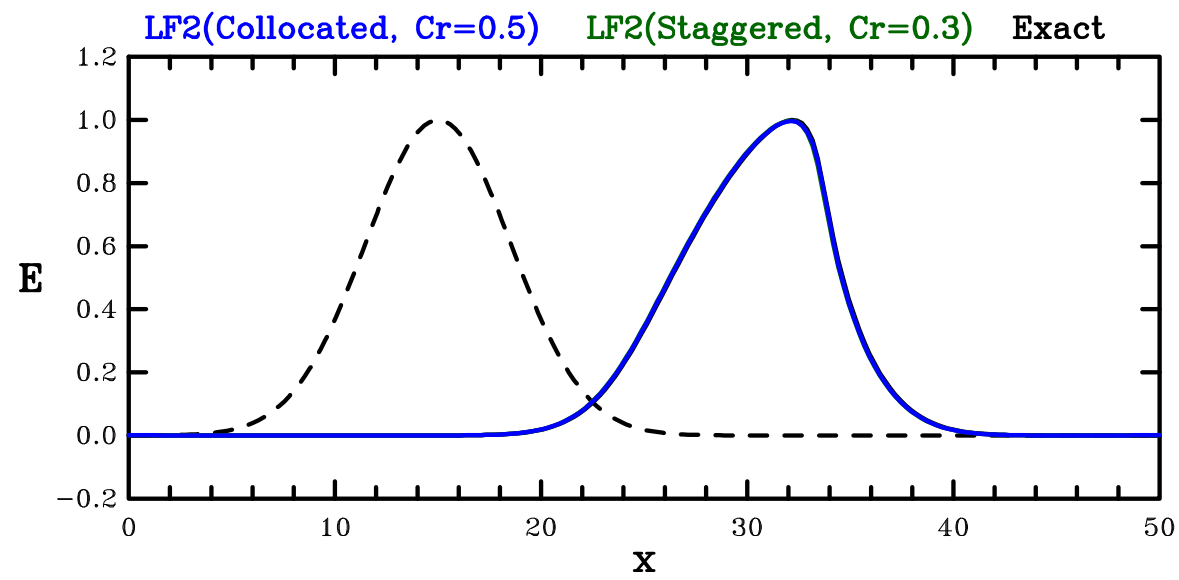

Fig. 11. Comparison of the exact and simulated electric field profiles for electromagnetic propagation in Kerr-nonlinear dielectric material at the normalized time $t=15$. The numerical solutions are computed with the LF2 scheme within collocated $(C r=0.5$, blue $)$ and staggered $(C r=0.3$, green $)$ spatial grids. The parameters of the simulations are $\gamma=0.1$ and $s=\nu \Delta t / \Delta x^{4}=0.004$. The initial profile (dashed) is also shown. The exact and simulated profiles, which are superimposed for $t=15$, are indistinguishable. 

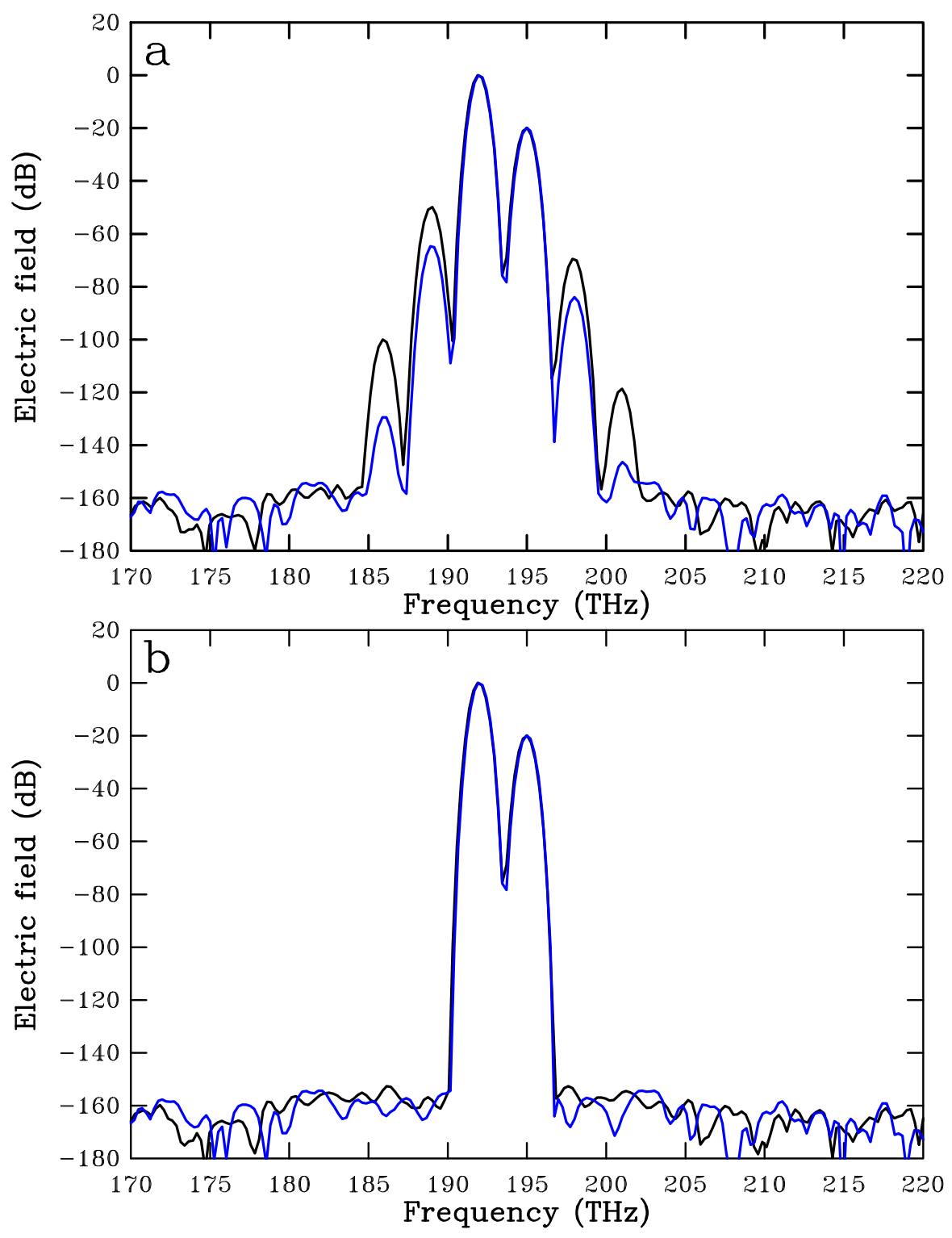

Fig. 12. Normalized power spectral density for two-dimensional electric field computations (blue curves) employing the LF2 scheme within the nonlinear optical medium presented in Fig. 13. The signal used in the spectra is detected at $x=10 \mu \mathrm{m}$ and $y=0 \mu \mathrm{m}$ for $E_{p}=5.1 \times 10^{7} \mathrm{~V} / \mathrm{m}$ using values of a) $\chi^{(3)}=1.0 \times 10^{-18} \mathrm{~m}^{2} / \mathrm{V}^{2}$ and b) $\chi^{(3)}=0 \mathrm{~m}^{2} / \mathrm{V}^{2}$. The normalized power spectra corresponding to plane waves are also superimposed (black curves). The spectra use a Kaizer window. 


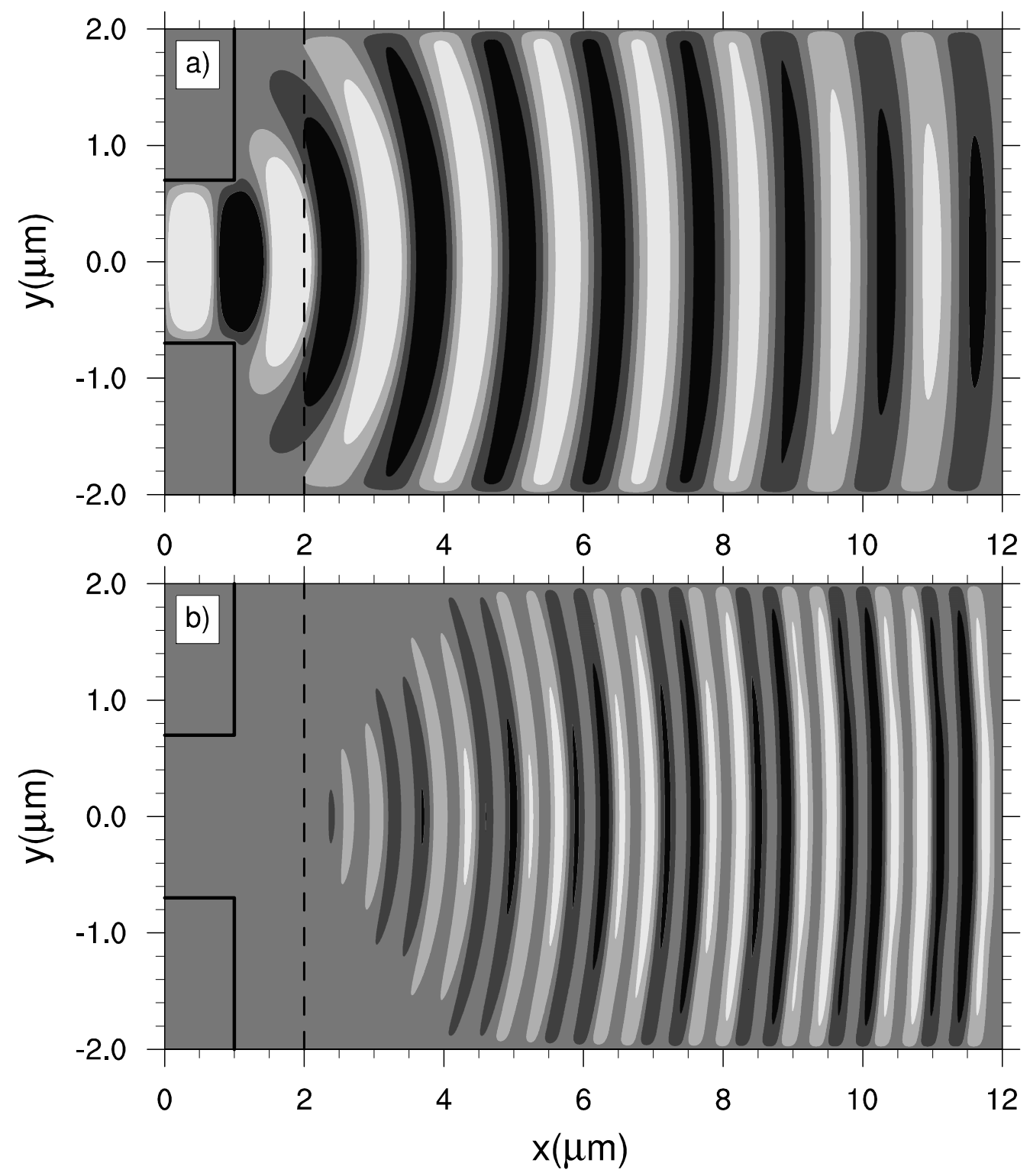

Fig. 13. Electric field computed by the LF2 scheme within a two dimensional structure. (a) Nonlinear simulation; and (b) difference between the nonlinear and linear simulations. The nonlinear structure consists of a dielectric slab of width $d=1.4$ $\mu \mathrm{m}$ at $x<1 \mu \mathrm{m}$ and a region of width $d=4 \mu \mathrm{m}$ at $x>1 \mu \mathrm{m}$. The dashed line at $x=2 \mu \mathrm{m}$ separates the medium into a linear region to the left $\left(\chi^{(3)}=0\right)$ and a nonlinear region to the right, $\left(\chi^{(3)}=1.0 \times 10^{-18} \mathrm{~m}^{2} / \mathrm{V}^{2}\right)$. The simulations employ a pump wave with amplitude $E_{p}=5.1 \times 10^{7} \mathrm{~V} / \mathrm{m}$. The field in Fig. $13 \mathrm{~b}$ is amplified by a factor of 100 . Converted waves are observed only in the nonlinear region demonstrating the FWM effect. 